\title{
Fractional magnetic Schrödinger-Kirchhoff problems with convolution and critical nonlinearities
}

\author{
Sihua Liang $^{a}$, Dušan D. Repovšs ${ }^{b, c}$, and Binlin Zhang ${ }^{d}$ \\ ${ }^{a}$ College of Mathematics, Changchun Normal University, Changchun, 130032, P.R. China \\ ${ }^{b}$ University of Ljubljana, Ljubljana, 1000, Slovenia \\ ${ }^{c}$ Institute of Mathematics, Physics and Mechanics, Ljubljana, 1000, Slovenia \\ ${ }^{d}$ College of Mathematics and System Science, Shandong University of Science and Technology, \\ Qingdao, 266590, P.R. China
}

\begin{abstract}
In this paper we are concerned with the existence and multiplicity of solutions for the fractional Choquardtype Schrödinger-Kirchhoff equations with electromagnetic fields and critical nonlinearity:

$$
\left\{\begin{array}{l}
\varepsilon^{2 s} M\left([u]_{s, A}^{2}\right)(-\Delta)_{A}^{s} u+V(x) u=\left(|x|^{-\alpha} * F\left(|u|^{2}\right)\right) f\left(|u|^{2}\right) u+|u|^{2_{s}^{*}-2} u, \quad x \in \mathbb{R}^{N} \\
u(x) \rightarrow 0, \quad \text { as }|x| \rightarrow \infty
\end{array}\right.
$$

where $(-\Delta)_{A}^{s}$ is the fractional magnetic operator with $0<s<1,2_{s}^{*}=2 N /(N-2 s), \alpha<\min \{N, 4 s\}$, $M: \mathbb{R}_{0}^{+} \rightarrow \mathbb{R}_{0}^{+}$is a continuous function, $A: \mathbb{R}^{N} \rightarrow \mathbb{R}^{N}$ is the magnetic potential, $F(|u|)=\int_{0}^{|u|} f(t) d t$, and $\varepsilon>0$ is a positive parameter. The electric potential $V \in C\left(\mathbb{R}^{N}, \mathbb{R}_{0}^{+}\right)$satisfies $V(x)=0$ in some region of $\mathbb{R}^{N}$, which means that this is the critical frequency case. We first prove the $(P S)_{c}$ condition, by using the fractional version of the concentration compactness principle. Then, applying also the mountain pass theorem and the genus theory, we obtain the existence and multiplicity of semiclassical states for the above problem. The main feature of our problems is that the Kirchhoff term $M$ can vanish at zero.
\end{abstract}

Keywords: Fractional magnetic operator; Choquard-type equation; Critical nonlinearity; Variational method.

2010 Mathematics Subject Classification: 35J10; 35B99; 35J60; 47G20.

\section{Introduction and main results}

In this paper we consider the fractional Choquard-Kirchhoff type problem with electromagnetic fields and critical nonlinearity:

$$
\left\{\begin{array}{l}
\varepsilon^{2 s} M\left([u]_{s, A}^{2}\right)(-\Delta)_{A}^{s} u+V(x) u=\left(\mathcal{K}_{\alpha} * F\left(|u|^{2}\right)\right) f\left(|u|^{2}\right) u+|u|^{2_{s}^{*}-2} u, x \in \mathbb{R}^{N}, \\
u(x) \rightarrow 0, \quad \text { as }|x| \rightarrow \infty
\end{array}\right.
$$

where $\varepsilon>0$ is a positive parameter, $N>2 s, 0<s<1,2_{s}^{*}=2 N /(N-2 s)$ is the critical Sobolev exponent, $V \in C\left(\mathbb{R}^{N}, \mathbb{R}_{0}^{+}\right)$is an electric potential, $\mathcal{K}_{\alpha}(x)=|x|^{-\alpha}, \alpha<\min \{N, 4 s\}, A \in C\left(\mathbb{R}^{N}, \mathbb{R}^{N}\right)$ is a magnetic potential, and

$$
[u]_{s, A}^{2}:=\iint_{\mathbb{R}^{2 N}} \frac{\left|u(x)-e^{i(x-y) \cdot A\left(\frac{x+y}{2}\right)} u(y)\right|^{2}}{|x-y|^{N+2 s}} d x d y
$$


If $A$ is a smooth function, the fractional operator $(-\Delta)_{A}^{S}$, which up to normalization constants can be defined on smooth functions $u$ as

$$
(-\Delta)_{A}^{s} u(x):=2 \lim _{\varepsilon \rightarrow 0} \int_{\mathbb{R}^{N} \backslash B_{\varepsilon}(x)} \frac{u(x)-e^{i(x-y) \cdot A\left(\frac{x+y}{2}\right)} u(y)}{|x-y|^{N+2 s}} d y, \quad x \in \mathbb{R}^{N},
$$

has recently been introduced in [11]. Hereafter, $B_{\varepsilon}(x)$ denotes the ball of $\mathbb{R}^{N}$ centered at $x \in \mathbb{R}^{N}$ and of radius $\varepsilon>0$. For details on fractional magnetic operators we refer to [11], and for the physical background we refer to $[16,17,18]$.

This paper was motivated by some works concerning the magnetic Schrödinger equation

$$
-(\nabla u-i A)^{2} u+V(x) u=f(x,|u|) u,
$$

which have appeared in recent years (see $[4,10,12,19,33])$ and have extensively studied 1.2 , when the above magnetic operator is defined as

$$
-(\nabla u-i A)^{2} u=-\Delta u+2 i A(x) \cdot \nabla u+|A(x)|^{2} u+i u \operatorname{div} A(x) .
$$

As stated in [34], up to correcting the operator by the factor $(1-s)$, it follows that $(-\Delta)_{A}^{s} u$ converges to $-(\nabla u-i A)^{2} u$ as $s \rightarrow 1$.

Thus, up to normalization, the nonlocal case can be seen as an approximation of the local one. The motivation for its introduction was described in $[11,34]$ and relies essentially on the Lévy-Khintchine formula for the generator of a general Lévy process. If the magnetic field $A \equiv 0$, the operator $(-\Delta)_{A_{\varepsilon}}^{s}$ can be reduced to the fractional Laplacian operator $(-\Delta)^{s}$, which may be viewed as the infinitesimal generator of a Lévy stable diffusion processes [3]. This operator arises in the description of various phenomena in applied sciences, such as phase transitions, materials science, conservation laws, minimal surfaces, water waves, optimization, plasma physics, etc., see [13] and references therein.

The study of fractional and nonlocal operators of elliptic type has recently attracted a lot of attention. For the cases in which bounded domains and the entire space are involved, we refer the readers e.g. to [1, 7, 25, 27, 40, 41] and the references therein. When the interaction between the particles is considered, i.e., when the nonlinear term $f(u)$ is of type $\left(\mathcal{K}_{\alpha} *|u|^{p}\right)|u|^{p-2} u$, this type of problem is usually called the Choquard-type equation and has been investigated by many authors, see e.g. [23, 35].

Another strong motivation for studying problem (1.1) is the significant feature of Kirchhoff-type problems. More precisely, in 1883 Kirchhoff proposed the following model

$$
\rho \frac{\partial^{2} u}{\partial t^{2}}-\left(\frac{p_{0}}{\lambda}+\frac{Y}{2 L} \int_{0}^{L}\left|\frac{\partial u}{\partial x}\right|^{2} d x\right) \frac{\partial^{2} u}{\partial x^{2}}=0
$$

as a generalization of the well-known D'Alembert's wave equation for free vibrations of elastic strings. Here, $L$ is the length of the string, $\lambda$ is the area of the cross section, $Y$ is the Young modulus of the material, $\rho$ is the mass density, and $p_{0}$ is the initial tension. Essentially, Kirchhoff's model takes into account the changes in the length of the string produced by transverse vibrations. For recent results in this direction, we refer the reader e.g. to $[21,22]$.

Recently, Fiscella and Valdinoci [15] first deduced a stationary fractional Kirchhoff model which considered the nonlocal aspect of the tension arising from nonlocal measurements of the fractional length of the string (see [15, Appendix] for more details). More precisely, the following Kirchhoff-type problem involving critical exponent was studied in [15]:

$$
\begin{cases}M\left([u]_{s}^{2}\right)(-\Delta)^{s} u=\lambda f(x, u)+|u|^{2_{s}^{*}-2} u & \text { in } \Omega \\ u=0 & \text { in } \mathbb{R}^{N} \backslash \Omega .\end{cases}
$$

where $\Omega$ is an open bounded domain in $\mathbb{R}^{N}$. By using the mountain pass theorem and the concentration compactness principle, together with a truncation technique, the existence of non-negative solutions for problem (1.4) was obtained. 
Here we point out that $M(0)>0$ in (1.4), this is called the non-degenerate case. Otherwise, the problem is called degenerate if $M(0)=0$. In recent years, there has been a lot of interest in studying fractional Kirchhofftype problems, here we just list some references, e.g., see [20, 27, 29] for recent results on the non-degenerate case, $[5,30,35,38,39]$ for recent results on the degenerate case, and [24, 31] for discussions of both cases.

Next, let us mention some enlightening works related to problem (1.1). Mingqi et al. [24] first studied the following Schrödinger-Kirchhoff type equation involving the fractional $p$-Laplacian and the magnetic operator

$$
M\left([u]_{s, A}^{2}\right)(-\Delta)_{A}^{s} u+V(x) u=f(x,|u|) u \quad \text { in } \mathbb{R}^{N}
$$

where the right-hand term in (1.5) satisfies the subcritical growth. By using variational methods, they obtained several existence results for problem (1.5). Using similar methods, for $M(t)=a+b t$ with $a \in \mathbb{R}_{0}^{+}$and $b \in \mathbb{R}^{+}$, Wang and Xiang [35] proved the existence of two solutions and infinitely many solutions for fractional SchrödingerChoquard-Kirchhoff type equations with external magnetic operator and critical exponent in the sense of the Hardy-Littlewood-Sobolev inequality.

Binlin et al. [8] first considered the following singularly perturbed fractional Schrödinger equations:

$$
\varepsilon^{2 s}(-\Delta)_{A_{\varepsilon}}^{s} u+V(x) u=f(x,|u|) u+K(x)|u|^{2_{\alpha}^{*}-2} u \quad \text { in } \mathbb{R}^{N},
$$

where $V(x)$ satisfies some assumptions. By using variational methods, they proved the existence of solutions $u_{\varepsilon}$ which tends to the trivial solution as $\varepsilon \rightarrow 0$. Moreover, they proved the existence of infinite many solutions and sign-changing solutions for problem (1.6) without magnetic field under some additional assumptions.

Subsequently, Liang et al. [20] investigated the existence and multiplicity of solutions for problem (1.1) without Choquard-type term in the non-degenerate Kirchhoff case. Very recently, by employing variational methods, Ambrosio [2] obtained the existence and concentration of nontrivial solutions for a singularly perturbed fractional Choquard problem with a subcritical nonlinearity and an external magnetic field.

Inspired by the above works, in particular $[8,14,20,24]$, we consider in this article the existence and multiplicity of solutions for the fractional Choquard-type problems with electromagnetic fields and critical nonlinearity in the possibly degenerate Kirchhoff context. It is worthwhile to remark that in the arguments developed in [8, 14], one of the key points is to prove the $(P S)_{c}$ condition. Here we use the fractional version of Lions' second concentration compactness principle and concentration compactness principle at infinity to prove that the $(P S)_{c}$ condition holds, which is different from methods used in [8, 14].

In fact, the appearance of the magnetic field also brings additional difficulties into the study of our problem, e.g., the effects of the magnetic fields on the linear spectral sets and on the solution structure, and the possible interactions between the magnetic fields and the linear potentials. Therefore, we need to develop new techniques to conquer difficulties induced by these new features as well as the possibly degenerate nature of the Kirchhoff coefficient.

Suppose that functions $V(x), M(t)$ and $f(t)$ satisfy the following conditions:

$(V) V(x) \in C\left(\mathbb{R}^{N}, \mathbb{R}\right), \min _{x \in \mathbb{R}^{N}} V(x)=0$ and there is $\tau_{0}>0$ such that the set $V^{\tau_{0}}=\left\{x \in \mathbb{R}^{N}: V(x)<\tau_{0}\right\}$ has finite Lebesgue measure.

$(M)\left(M_{1}\right)$ there exists $\sigma \in\left(1,2_{s}^{*} / 2\right)$ satisfying $\sigma \widetilde{M}(t) \geq M(t) t$ for all $t \geq 0$, where $\widetilde{M}(t)=\int_{0}^{t} M(s) d s$; $\left(M_{2}\right)$ there exists $m_{1}>0$ such that $M(t) \geq m_{1} t^{\sigma-1}$ for all $t \in \mathbb{R}^{+}$and $M(0)=0$.

$(F)\left(f_{1}\right) f \in C\left(\mathbb{R}^{+}, \mathbb{R}\right)$

$\left(f_{2}\right)$ there exist $c_{0}>0$ and $\max \{\sigma, 2\}<p<2_{s}^{*}$ such that $|f(t)| \leq c_{0}|t|^{\frac{p-1}{2}}$;

$\left(f_{3}\right)$ there exist $2 \sigma<\mu<2_{s}^{*}$ such that $0<\mu F(t) \leq f(t) t$ for all $t \in \mathbb{R}^{+}$, where $F(t)=\int_{0}^{t} f(s) d s$.

The following is our first main result, the existence theorem for problem (1.1).

Theorem 1.1. Let the conditions $(V),(M)$ and $(F)$ be satisfied. Then for any $\kappa>0$, there is $\mathcal{E}_{\kappa}>0$ such that if $0<\varepsilon<\mathcal{E}_{\kappa}$, then problem (1.1) has at least one solution $u_{\varepsilon}$ satisfying

$$
\frac{2 \mu-\sigma}{4 \sigma} \iint_{\mathbb{R}^{2 N}} \frac{F\left(\left|u_{\varepsilon}(x)\right|^{2}\right) F\left(\left|u_{\varepsilon}(y)\right|^{2}\right)}{|x-y|^{\alpha}} d x d y+\left(\frac{1}{2 \sigma}-\frac{1}{2_{s}^{*}}\right) \int_{\mathbb{R}^{N}}\left|u_{\varepsilon}\right|^{2_{s}^{*}} d x \leq \kappa \varepsilon^{\frac{2 s 2_{s}^{*}}{2 *}-4 \sigma},
$$




$$
\left(\frac{1}{2 \sigma}-\frac{1}{\mu}\right) \alpha_{0} \varepsilon^{2 s}\left[u_{\varepsilon}\right]_{s, A}^{2 \sigma}+\left(\frac{1}{2}-\frac{1}{\mu}\right) \int_{\mathbb{R}^{N}} V(x)\left|u_{\varepsilon}\right|^{2} d x \leq \kappa \varepsilon^{\frac{2 s_{s}^{2}}{2_{s}^{*}-4 \sigma}} .
$$

Moreover, $u_{\varepsilon} \rightarrow 0$ in $E$ as $\varepsilon \rightarrow 0$.

The following is our second main result, the multiplicity theorem for problem (1.1).

Theorem 1.2. Let the conditions $(V),(M)$ and $(F)$ be satisfied. Then for any $m \in \mathbb{N}$ and $\kappa>0$, there is $\mathcal{E}_{m \kappa}>0$ such that if $0<\varepsilon<\mathcal{E}_{m \kappa}$, then problem (1.1) has at least $m$ pairs of solutions $u_{\varepsilon, i}, u_{\varepsilon,-i}, i=1,2, \cdots, m$ which satisfy estimates (1.7) and (1.8). Moreover, $u_{\varepsilon, i} \rightarrow 0$ in $E$ as $\varepsilon \rightarrow 0, i=1,2, \cdots, m$.

\section{Functional setting}

In this paper, we shall use Banach space $E$ defined by

$$
E=\left\{u \in H_{A}^{s}\left(\mathbb{R}^{N}, \mathbb{C}\right): \int_{\mathbb{R}^{N}} V(x)|u|^{2} d x<\infty\right\}
$$

with the norm

$$
\|u\|_{E}:=\left([u]_{s, A}^{2}+\int_{\mathbb{R}^{N}} V(x)|u|^{2} d x\right)^{\frac{1}{2}},
$$

where $V$ is non-negative, $H_{A_{\varepsilon}}^{s}\left(\mathbb{R}^{N}, \mathbb{C}\right)$ is the fractional Sobolev space defined by

$$
H_{A}^{s}\left(\mathbb{R}^{N}, \mathbb{C}\right)=\left\{u \in L^{2}\left(\mathbb{R}^{N}, \mathbb{C}\right):[u]_{s, A}<\infty\right\},
$$

where $s \in(0,1)$ and $[u]_{s, A}$ denotes the so-called Gagliardo semi-norm, that is

$$
[u]_{s, A}=\left(\iint_{\mathbb{R}^{2 N}} \frac{\left|u(x)-e^{i(x-y) \cdot A\left(\frac{x+y}{2}\right)} u(y)\right|^{2}}{|x-y|^{N+2 s}} d x d y\right)^{1 / 2}
$$

and $H_{A}^{s}\left(\mathbb{R}^{N}, \mathbb{C}\right)$ is endowed with the norm

$$
\|u\|_{H_{A}^{s}\left(\mathbb{R}^{N}, \mathbb{C}\right)}=\left([u]_{s, A}^{2}+\|u\|_{L^{2}}^{2}\right)^{\frac{1}{2}} .
$$

By assumption $(V)$, we know that the embedding $E \hookrightarrow H_{A}^{s}\left(\mathbb{R}^{N}, \mathbb{C}\right)$ is continuous. Note that the norm $\|\cdot\|_{E}$ is equivalent to the norm $\|\cdot\|_{\varepsilon}$ defined by

$$
\|u\|_{\varepsilon}:=\left([u]_{s, A}^{2}+\varepsilon^{-2 s} \int_{\mathbb{R}^{N}} V(x)|u|^{2} d x\right)^{\frac{1}{2}}
$$

for each $\varepsilon>0$. It is obvious that for each $\theta \in\left[2,2_{s}^{*}\right]$, there is $c_{\theta}>0$, independent of $0<\varepsilon<1$, such that

$$
|u|_{\theta} \leq c_{\theta}\|u\|_{E} \leq c_{\theta}\|u\|_{\varepsilon} .
$$

Hereafter, we shortly denote by $\|\cdot\|_{\nu}$ the norm of Lebesgue space $L^{\nu}(\Omega)$ with $\nu \geq 1$.

We first recall the following embedding theorem:

Proposition 2.1. (see [11, Lemma 3.5]). Let $A \in C\left(\mathbb{R}^{N}, \mathbb{R}^{N}\right)$. Then the embedding

$$
H_{A}^{s}\left(\mathbb{R}^{N}, \mathbb{C}\right) \hookrightarrow L^{\theta}\left(\mathbb{R}^{N}, \mathbb{C}\right),
$$

is continuous for any $\theta \in\left[2,2_{s}^{*}\right]$. Moreover, the embedding

$$
H_{A}^{s}\left(\mathbb{R}^{N}, \mathbb{C}\right) \hookrightarrow L_{\mathrm{loc}}^{\theta}\left(\mathbb{R}^{N}, \mathbb{C}\right)
$$

is compact for any $\theta \in\left[1,2_{s}^{*}\right)$. 
We shall use the following diamagnetic inequality:

Lemma 2.1. (see $\left[11\right.$, Lemma 3.3]). For every $u \in H_{A}^{s}\left(\mathbb{R}^{N}, \mathbb{C}\right)$,

$$
|u| \in H^{s}\left(\mathbb{R}^{N}\right)
$$

More precisely,

$$
[|u|]_{s} \leq[u]_{s, A}
$$

By Proposition 3.6 in [13], we have

$$
[u]_{s}=\left\|(-\Delta)^{\frac{s}{2}}\right\|_{L^{2}\left(\mathbb{R}^{N}\right)}
$$

for any $u \in H^{s}\left(\mathbb{R}^{N}\right)$, i.e.

$$
\iint_{\mathbb{R}^{2 N}} \frac{|u(x)-u(y)|^{2}}{|x-y|^{N+2 s}} d x d y=\int_{\mathbb{R}^{N}}\left|(-\Delta)^{\frac{s}{2}} u(x)\right|^{2} d x
$$

Thus

$$
\iint_{\mathbb{R}^{2 N}} \frac{(u(x)-u(y))(v(x)-v(y))}{|x-y|^{N+2 s}} d x d y=\int_{\mathbb{R}^{N}}(-\Delta)^{\frac{s}{2}} u(x) \cdot(-\Delta)^{\frac{s}{2}} v(x) d x .
$$

To obtain the solution of problem (1.1), we shall use the following equivalent form

$$
\left\{\begin{array}{l}
M\left([u]_{s, A}^{2}\right)(-\Delta)_{A}^{s} u+\varepsilon^{-2 s} V(x) u=\varepsilon^{-2 s} \int_{\mathbb{R}^{N}} \frac{F\left(|u|^{2}\right)}{|x-y|^{\alpha}} d y f\left(|u|^{2}\right) u+\varepsilon^{-2 s}|u|^{2_{s}^{*}-2} u, x \in \mathbb{R}^{N}, \\
u(x) \rightarrow 0, \quad \text { as } \quad|x| \rightarrow \infty
\end{array}\right.
$$

for $\varepsilon \rightarrow 0$.

The energy functional $J_{\varepsilon}: E \rightarrow \mathbb{R}$ associated with problem (2.2)

$$
J_{\varepsilon}(u):=\frac{1}{2} \widetilde{M}\left([u]_{s, A}^{2}\right)+\frac{\varepsilon^{-2 s}}{2} \int_{\mathbb{R}^{N}} V(x)|u|^{2} d x-\frac{\varepsilon^{-2 s}}{4} \iint_{\mathbb{R}^{2 N}} \frac{F\left(|u(x)|^{2}\right) F\left(|u(y)|^{2}\right)}{|x-y|^{\alpha}} d x d y-\frac{\varepsilon^{-2 s}}{2_{s}^{*}} \int_{\mathbb{R}^{N}}|u|^{2_{s}^{*}} d x
$$

is well defined. Under the assumptions, it is easy to check that as shown in $[32,36], J_{\varepsilon} \in C^{1}(E, \mathbb{R})$ and its critical points are weak solutions of problem (2.2).

By condition $\left(f_{2}\right)$, we have

$$
F\left(|u|^{2}\right) \leq C\left(|u|^{2}+|u|^{p}\right), \quad \text { for all } u \in H_{A}^{s}\left(\mathbb{R}^{N}, \mathbb{C}\right) .
$$

Note that, by the Hardy-Littlewood-Sobolev inequality, the integral

$$
\iint_{\mathbb{R}^{2 N}} \frac{F\left(|u(x)|^{2}\right) F\left(|u(y)|^{2}\right)}{|x-y|^{\alpha}} d x d y
$$

is well defined if $F\left(|u|^{2}\right) \in L^{r}\left(\mathbb{R}^{N}\right)$ for some $r>1$ satisfying

$$
\frac{2}{r}+\frac{\alpha}{N}=2
$$

that is $r=2 N /(2 N-\alpha)$. Actually, by $\alpha<\min \{N, 4 s\}$, it follows that $2<2 r<2_{s}^{*}$. Moreover, from $2<p r<2_{s}^{*}$, we can deduce

$$
\begin{aligned}
\int_{\mathbb{R}^{N}}\left|F\left(|u|^{2}\right)\right|^{r} d x & \leq 2^{r-1} C^{r}\left(\int_{\mathbb{R}^{N}}|u|^{2 r} d x+\int_{\mathbb{R}^{N}}|u|^{p r} d x\right) \\
& \leq 2^{r-1} C^{r}\left(C_{2 r}^{2 r}\|u\|^{2 r}+C_{p r}^{p r}\|u\|^{p r}\right) \quad \text { for all } u \in H_{A}^{s}\left(\mathbb{R}^{N}, \mathbb{C}\right)
\end{aligned}
$$


By a standard argument, one can show that $J_{\varepsilon}(u)$ is of class $C^{1}$ and

$$
\begin{aligned}
\left\langle J_{\varepsilon}^{\prime}(u), v\right\rangle= & M\left([u]_{s, A}^{2}\right) \operatorname{Re} \iint_{\mathbb{R}^{2 N}} \frac{\left(u(x)-e^{i(x-y) \cdot A\left(\frac{x+y}{2}\right)} u(y)\right) \overline{\left(v(x)-e^{i(x-y) \cdot A\left(\frac{x+y}{2}\right)} v(y)\right)}}{|x-y|^{N+2 s}} d x d y \\
& +\varepsilon^{-2 s} \operatorname{Re} \int_{\mathbb{R}^{N}} V(x) u \bar{v} d x-\varepsilon^{-2 s} \operatorname{Re} \int_{\mathbb{R}^{N}}\left(\mathcal{K}_{\mu} * F\left(|u|^{2}\right)\right) f\left(|u|^{2}\right) u \bar{v} d x-\varepsilon^{-2 s} \operatorname{Re} \int_{\mathbb{R}^{N}}|u|^{2_{s}^{*}-2} u \bar{v} d x,
\end{aligned}
$$

for all $u, v \in E$. Hence a critical point of $J_{\varepsilon}$ is a weak solution of problem (1.1).

Now we recall the general version of the mountain pass theorem in [32] which will be used later.

Theorem 2.1. Let $\mathcal{J}$ be a functional on a Banach space $Y$ and $\mathcal{J} \in C^{1}(Y, \mathbb{R})$. Let us assume that there exist $\zeta, \rho>0$ such that

(i) $\mathcal{J}(u) \geq \zeta$, for every $u \in Y$ with $\|u\|=\rho$;

(ii) $\mathcal{J}(0)=0$ and $\mathcal{J}(e)<\zeta$ for some $e \in Y$ with $\|e\|>\rho$.

Let us define $\Gamma=\{\gamma \in C([0,1] ; Y): \gamma(0)=0, \gamma(1)=e\}$ and

$$
c=\inf _{\gamma \in \Gamma} \max _{t \in[0,1]} \mathcal{J}(\gamma(t)) .
$$

Then there exists a sequence $\left\{u_{n}\right\}_{n} \subset Y$ such that $\mathcal{J}\left(u_{n}\right) \rightarrow c$ and $\mathcal{J}^{\prime}\left(u_{n}\right) \rightarrow 0$ in $Y^{\prime}$ (dual of $Y$ ).

By the assumptions $(V),(M)$ and $(F)$, one can see that $J_{\varepsilon}(u)$ has the mountain pass geometry.

Lemma 2.2. Assume that conditions $(V),(M)$ and $(F)$ hold. Then the functional $J_{\varepsilon}$ satisfies the conclusions (i)-(ii) of Theorem 2.1.

Proof. For each $\varepsilon>0$, by the fractional Sobolev embedding, $\left(M_{2}\right)$ and $\left(f_{2}\right)$, we have

$$
\begin{aligned}
J_{\varepsilon}(u) & :=\frac{1}{2} \widetilde{M}\left([u]_{s, A}^{2}\right)+\frac{\varepsilon^{-2 s}}{2} \int_{\mathbb{R}^{N}} V(x)|u|^{2} d x-\frac{\varepsilon^{-2 s}}{4} \iint_{\mathbb{R}^{2 N}} \frac{F\left(|u(x)|^{2}\right) F\left(|u(y)|^{2}\right)}{|x-y|^{\alpha}} d x d y-\frac{\varepsilon^{-2 s}}{2_{s}^{*}} \int_{\mathbb{R}^{N}}|u|^{2_{s}^{*}} d x \\
& \geq \min \left\{\frac{m_{1}}{2 \sigma}, \frac{1}{2}\right\}\|u\|_{\varepsilon}^{2 \sigma}-\varepsilon^{-2 s} C\|u\|_{\varepsilon}^{2 p}-\frac{\varepsilon^{-2 s}}{2_{s}^{*}} S^{\frac{-2_{s}^{*}}{2}}\|u\|_{\varepsilon}^{2_{s}^{*}},
\end{aligned}
$$

for all $u \in E$. It follows from $\max \{2, \sigma\}<p$ that there exist small enough $\varrho_{\varepsilon}>0$ and $\alpha_{\varepsilon}>0$ such that $J_{\varepsilon}(u) \geq \alpha_{\varepsilon}>0$ for all $u \in E$ with $\|u\|_{\varepsilon}=\varrho_{\varepsilon}$, and all $\varepsilon>0$. Hence (i) in Theorem 2.1 holds.

Now we verify condition $(i i)$ in Theorem 2.1. Let $\varphi_{0} \in C_{0}^{\infty}\left(\mathbb{R}^{N}, \mathbb{C}\right)$ with $\left\|\varphi_{0}\right\|_{\varepsilon}=1$. By $\left(M_{2}\right)$, we have

$$
\widetilde{M}(t) \leq \widetilde{M}(1) t^{\sigma} \text { for all } t \geq 1 \text {. }
$$

Then by $\left(f_{3}\right)$, the following holds

$$
\begin{aligned}
J_{\varepsilon}\left(t \varphi_{0}\right) & \leq \widetilde{M}(1) t^{2 \sigma}+\frac{1}{2} t^{2}-\frac{\varepsilon^{-2 s}}{4} \int_{\mathbb{R}^{N}}\left(\mathcal{K}_{\alpha} * F\left(\left|t \varphi_{0}\right|^{2}\right)\right) F\left(\left|t \varphi_{0}\right|^{2}\right) d x-\frac{\varepsilon^{-2 s}}{2_{s}^{*}} t^{2_{s}^{*}}\left|\varphi_{0}\right|_{2_{s}^{*}}^{2_{s}^{*}} \\
& \leq \widetilde{M}(1) t^{2 \sigma}+\frac{1}{2} t^{2}-\frac{\varepsilon^{-2 s}}{2_{s}^{*}} t^{2_{s}^{*}}\left|\varphi_{0}\right|_{2_{s}^{*}}^{2_{s}^{*}},
\end{aligned}
$$

and hence $J_{\varepsilon}\left(t \varphi_{0}\right) \rightarrow-\infty$ as $t \rightarrow \infty$, since $2 \sigma<2_{s}^{*}$. Therefore, there exists large enough $t_{0}$ such that $J_{\varepsilon}\left(t_{0} \varphi_{0}\right)<0$. Then we take $e=t_{0} \varphi_{0}$ and $J_{\varepsilon}(e)<0$. Hence $(i i)$ in Theorem 2.1 holds. The proof is thus complete. 


\section{Verification of $(P S)_{c}$ condition}

In this section we recall the fractional version of concentration compactness principle in the fractional Sobolev space, see [28, 39, 43] for more details.

Lemma 3.1. (see [28, Theorem 1.5]) Let $\Omega \subseteq \mathbb{R}^{N}$ be an open subset and let $\left\{u_{n}\right\}_{n}$ be a sequence in $H^{s}\left(\mathbb{R}^{N}\right)$, weakly converging to $u$ as $n \rightarrow \infty$ and such that $\left|u_{n}\right|^{2_{s}^{*}} \rightarrow \nu$ and $\left|(-\Delta)^{\frac{s}{2}} u_{n}\right|^{2} \rightarrow \mu$ in the sense of measures. Then either $u_{n} \rightarrow u$ in $L_{\text {loc }}^{2_{s}^{*}}\left(\mathbb{R}^{N}\right)$ or there exist a (at most countable) set of distinct points $\left\{x_{j}\right\}_{j \in I} \subseteq \bar{\Omega}$ and positive numbers $\left\{\nu_{j}\right\}_{j \in I}$ such that

$$
\nu=|u|^{2_{s}^{*}}+\sum_{j \in I} \delta_{x_{j}} \nu_{j}, \quad \nu_{j}>0
$$

If, in addition, $\Omega$ is bounded, then there exist a positive measure $\widetilde{\mu} \in \mathcal{M}\left(\mathbb{R}^{N}\right)$ with supp $\widetilde{\mu} \subseteq \bar{\Omega}$ and positive numbers $\left\{\mu_{j}\right\}_{j \in I}$ such that

$$
\mu=\left|(-\Delta)^{\frac{s}{2}} u\right|^{2}+\widetilde{\mu}+\sum_{j \in I} \delta_{x_{j}} \mu_{j}, \quad \mu_{j}>0
$$

and

$$
\nu_{j} \leq\left(S^{-1} \mu\left(\left\{x_{j}\right\}\right)\right)^{\frac{2_{s}^{*}}{2}}
$$

where $S$ is the best Sobolev constant, i.e.

$$
S=\inf _{u \in H^{s}\left(\mathbb{R}^{N}\right)} \frac{\int_{\mathbb{R}^{N}}\left|(-\Delta)^{\frac{s}{2}} u\right|^{2} d x}{\int_{\mathbb{R}^{N}}|u|^{2_{s}^{*}} d x},
$$

$x_{j} \in \mathbb{R}^{N}, \delta_{x_{j}}$ are Dirac measures at $x_{j}$ and $\mu_{j}, \nu_{j}$ are constants.

In the case $\Omega=\mathbb{R}^{N}$, the above principle of concentration compactness does not provide any information about the possible loss of mass at infinity. The following result expresses this fact in quantitative terms.

Lemma 3.2. (see $\left[43\right.$, Lemma 3.5]) Let $\left\{u_{n}\right\}_{n} \subset H^{s}\left(\mathbb{R}^{N}\right)$ be such that $u_{n} \rightarrow u$ weakly converges in $H^{s}\left(\mathbb{R}^{N}\right)$, $\left|u_{n}\right|^{2_{s}^{*}} \rightarrow \nu$ and $\left|(-\Delta)^{\frac{s}{2}} u_{n}\right|^{2} \rightarrow \mu$ weakly-* converges in $\mathcal{M}\left(\mathbb{R}^{N}\right)$ and define

(i) $\mu_{\infty}=\lim _{R \rightarrow \infty} \limsup _{n \rightarrow \infty} \int_{\left\{x \in \mathbb{R}^{N}:|x|>R\right\}}\left|(-\Delta)^{\frac{s}{2}} u_{n}\right|^{2} d x$,

(ii) $\nu_{\infty}=\lim _{R \rightarrow \infty} \limsup _{n \rightarrow \infty} \int_{\left\{x \in \mathbb{R}^{N}:|x|>R\right\}}\left|u_{n}\right|^{2_{s}^{*}} d x$.

Then the quantities $\nu_{\infty}$ and $\mu_{\infty}$ exist and satisfy the following

(iii) $\limsup _{n \rightarrow \infty} \int_{\mathbb{R}^{N}}\left|(-\Delta)^{\frac{s}{2}} u_{n}\right|^{2} d x=\int_{\mathbb{R}^{N}} d \mu+\mu_{\infty}$,

(iv) $\limsup _{n \rightarrow \infty} \int_{\mathbb{R}^{N}}\left|u_{n}\right|^{2_{s}^{*}} d x=\int_{\mathbb{R}^{N}} d \nu+\nu_{\infty}$,

(v) $\nu_{\infty} \leq\left(S^{-1} \nu_{\infty}\right)^{\frac{2_{s}^{*}}{2}}$.

The main result of this section is the following compactness result: 
Lemma 3.3. Suppose that conditions $(V),(M)$ and $(F)$ hold. Let $\left\{u_{n}\right\}_{n} \subset E$ be a $(P S)_{c}$ sequence of functional $J_{\varepsilon}$, i.e.

$$
J_{\varepsilon}\left(u_{n}\right) \rightarrow c \quad \text { and } \quad J_{\varepsilon}^{\prime}\left(u_{n}\right) \rightarrow 0 \quad \text { in } E^{\prime}
$$

as $n \rightarrow \infty$, where $E^{\prime}$ is the dual of $E$. Then for any $0<\varepsilon<1, J_{\varepsilon}$ satisfies $(P S)_{c}$ condition, for all $c \in\left(0, \sigma_{0} \varepsilon^{\frac{8 s \sigma}{2 *-4 \sigma}}\right)$, where $\sigma_{0}:=\left(\frac{1}{\mu}-\frac{1}{2_{s}^{*}}\right)\left(m_{1} S^{2 \sigma}\right)^{\frac{2_{s}^{*}}{2_{s}^{*}-4 \sigma}}$, i.e. any $(P S)_{c}$-sequence $\left\{u_{n}\right\}_{n} \subset E$ has a strongly convergent subsequence in $E$.

Proof. If $\inf _{n \geq 1}\|u\|_{\varepsilon}=0$, then there exists a subsequence of $\left\{u_{n}\right\}_{n}$ (still denoted by $\left\{u_{n}\right\}_{n}$ ) such that $u_{n} \rightarrow 0$ in $E$ as $n \rightarrow \infty$. Thus, we assume that $d:=\inf _{n \geq 1}\|u\|_{\varepsilon}>0$ in the sequel.

By $J_{\varepsilon}\left(u_{n}\right) \rightarrow c$ and $J_{\varepsilon}^{\prime}\left(u_{n}\right) \rightarrow 0$ in $E^{\prime}$, there exists $C>0$ such that

$$
\begin{aligned}
c+o(1)\left\|u_{n}\right\|_{\varepsilon}= & J_{\varepsilon}\left(u_{n}\right)-\frac{1}{\mu}\left\langle J_{\varepsilon}^{\prime}\left(u_{n}\right), u_{n}\right\rangle=\frac{1}{2} \widetilde{M}\left(\left[u_{n}\right]_{s, A}^{2}\right)-\frac{1}{\mu} M\left(\left[u_{n}\right]_{s, A}^{2}\right)\left[u_{n}\right]_{s, A}^{2} \\
& +\left(\frac{1}{2}-\frac{1}{\mu}\right) \varepsilon^{-2 s} \int_{\mathbb{R}^{N}} V(x)\left|u_{n}\right|^{2} d x+\left(\frac{1}{\mu}-\frac{1}{2_{s}^{*}}\right) \varepsilon^{-2 s} \int_{\mathbb{R}^{N}}\left|u_{n}\right|^{2_{s}^{*}} d x \\
& +\varepsilon^{-2 s} \int_{\mathbb{R}^{N}}\left(\mathcal{K}_{\alpha} * F\left(\left|u_{n}\right|^{2}\right)\right)\left(\frac{1}{\mu} f\left(\left|u_{n}\right|^{2}\right)\left|u_{n}\right|^{2}-\frac{1}{4} F\left(\left|u_{n}\right|^{2}\right)\right) d x .
\end{aligned}
$$

It follows by $\left(M_{2}\right)$ and $\left(f_{3}\right)$ that

$$
\begin{aligned}
C+C\left\|u_{n}\right\|_{\varepsilon} & \geq\left(\frac{1}{2 \sigma}-\frac{1}{\mu}\right) M\left(\left[u_{n}\right]_{s, A}^{2}\right)\left[u_{n}\right]_{s, A}^{2}+\left(\frac{1}{2}-\frac{1}{\mu}\right) \varepsilon^{-2 s} \int_{\mathbb{R}^{N}} V(x)\left|u_{n}\right|^{2} d x \\
& \geq\left(\frac{1}{2 \sigma}-\frac{1}{\mu}\right) m_{1}\left[u_{n}\right]_{s, A}^{2 \sigma}+\left(\frac{1}{2}-\frac{1}{\mu}\right) \varepsilon^{-2 s} \int_{\mathbb{R}^{N}} V(x)\left|u_{n}\right|^{2} d x .
\end{aligned}
$$

This, together with $2<2 \sigma<2_{s}^{*}$, implies that $\left\{u_{n}\right\}_{n}$ is bounded in $E$. Furthermore, we can obtain $c \geq 0$ by passing to the limit in (3.1). Hence, by diamagnetic inequality, $\left\{\left|u_{n}\right|\right\}_{n}$ is bounded in $H^{s}\left(\mathbb{R}^{N}\right)$. Therefore for some subsequence, there is $u \in E$ such that $u_{n} \rightarrow u$ in $E$.

Since $2<p<\frac{2 N-\alpha}{N-2 s}<2_{s}^{*}$ and $2<\frac{4 N}{2 N-\alpha}<2_{s}^{*}$, by Proposition 2.1 we get that $\left|u_{n}\right| \rightarrow|u|$ strongly in $L^{\frac{2 N p}{2 N-\alpha}}\left(\mathbb{R}^{N}\right) \cap L^{\frac{4 N}{2 N-\alpha}}\left(\mathbb{R}^{N}\right)$. Hence the Brézis-Lieb Lemma implies that $u_{n} \rightarrow u$ strongly in $L^{\frac{2 N p}{2 N-\alpha}}\left(\mathbb{R}^{N}, \mathbb{C}\right) \cap$ $L^{\frac{4 N}{2 N-\alpha}}\left(\mathbb{R}^{N}, \mathbb{C}\right)$. By $\left(f_{2}\right)$, we have

$$
\begin{aligned}
\int_{\mathbb{R}^{N}}\left|F\left(\left|u_{n}\right|^{2}-F\left(|u|^{2}\right)\right)\right|^{\frac{2 N}{2 N-\alpha}} d x \leq & \left.\int_{\mathbb{R}^{N}}\left|f\left(|u|^{2}+\varrho\left(\left|u_{n}\right|^{2}-|u|^{2}\right)\right)\right|^{\frac{2 N}{2 N-\alpha}}|| u_{n}\right|^{2}-\left.|u|^{2}\right|^{\frac{2 N}{2 N-\alpha}} d x \\
\leq & \int_{\mathbb{R}^{N}}\left[C\left(1+\left(\left|u_{n}\right|+|u|\right)^{p-2}\right)\right]^{\frac{2 N}{2 N-\alpha}}\left(\left|u_{n}\right|+|u|\right)^{\frac{2 N}{2 N-\alpha}}\left|u_{n}-u\right|^{\frac{2 N}{2 N-\alpha}} d x \\
\leq & C^{\frac{2 N}{2 N-\alpha}} 2^{\frac{\alpha}{2 N-\alpha}} \int_{\mathbb{R}^{N}}\left(\left|u_{n}\right|+|u|\right)^{\frac{2 N}{2 N-\alpha}}\left|u_{n}-u\right|^{\frac{2 N}{2 N-\alpha}} d x \\
& +C^{\frac{2 N}{2 N-\alpha}} 2^{\frac{\alpha}{2 N-\alpha}} \int_{\mathbb{R}^{N}}\left(\left|u_{n}\right|+|u|\right)^{(p-1) \frac{2 N}{2 N-\alpha}}\left|u_{n}-u\right|^{\frac{2 N}{2 N-\alpha}} d x .
\end{aligned}
$$

Using the Hölder inequality, we can deduce

$$
\begin{aligned}
\int_{\mathbb{R}^{N}}\left|F\left(\left|u_{n}\right|^{2}-F\left(|u|^{2}\right)\right)\right|^{\frac{2 N}{2 N-\alpha}} d x \leq & C^{\frac{2 N}{2 N-\alpha}} 2^{\frac{\alpha}{2 N-\alpha}}\left\|\left(\left|u_{n}\right|+|u|\right)^{\frac{2 N}{2 N-\alpha}}\right\|_{L^{2}\left(\mathbb{R}^{N}\right)}\left\|\left|u_{n}-u\right|^{\frac{2 N}{2 N-\alpha}}\right\|_{L^{2}\left(\mathbb{R}^{N}\right)} \\
& +C^{\frac{2 N}{2 N-\alpha}} 2^{\frac{\alpha}{2 N-\alpha}}\left\|\left(\left|u_{n}\right|+|u|\right)^{(p-1) \frac{2 N}{2 N-\alpha}}\right\|_{L^{\frac{p}{p-1}}\left(\mathbb{R}^{N}\right)}\left\|\left|u_{n}-u\right|^{\frac{2 N}{2 N-\alpha}}\right\|_{L^{p}\left(\mathbb{R}^{N}\right)} \\
\leq & C\left\|\left|u_{n}-u\right|^{\frac{2 N}{2 N-\alpha}}\right\|_{L^{2}\left(\mathbb{R}^{N}\right)}+C\left\|\left|u_{n}-u\right|^{\frac{2 N}{2 N-\alpha}}\right\|_{L^{p}\left(\mathbb{R}^{N}\right)} \\
\rightarrow & 0,
\end{aligned}
$$


as $n \rightarrow \infty$, where $C>0$ is independent of $n$. Thus, we obtain that $F\left(\left|u_{n}\right|^{2} \rightarrow F\left(|u|^{2}\right)\right.$ in $L^{\frac{2 N}{2 N-\alpha}}\left(\mathbb{R}^{N}\right)$. Note that by the Hardy-Littlewood-Sobolev inequality, the Riesz potential defines a linear continuous map form $L^{\frac{2 N}{2 N-\alpha}}\left(\mathbb{R}^{N}\right)$ to $L^{\frac{2 N}{\alpha}}\left(\mathbb{R}^{N}\right)$. Then

$$
\mathcal{K}_{\alpha} * F\left(\left|u_{n}\right|^{2}\right) \rightarrow \mathcal{K}_{\alpha} * F\left(|u|^{2}\right) \quad \text { in } \quad L^{\frac{2 N}{\alpha}}\left(\mathbb{R}^{N}\right)
$$

as $n \rightarrow \infty$.

For $\varphi \in E$ fixed, by $\left(f_{2}\right)$ with $\varepsilon=1$ we have

$$
\begin{aligned}
\int_{\mathbb{R}^{N}}\left|f\left(\left|u_{n}\right|^{2}\right) u_{n} \bar{\varphi}\right|^{\frac{2 N}{2 N-\alpha}} d x \leq & C^{\frac{2 N}{2 N-\alpha}} 2^{\frac{\alpha}{2 N-\alpha}}\left(\int_{\mathbb{R}^{N}}\left(\left|u_{n}\right||\varphi|\right)^{\frac{2 N}{2 N-\alpha}} d x+\int_{\mathbb{R}^{N}}\left|u_{n}\right|^{(p-1) \frac{2 N}{2 N-\alpha}}|\varphi|^{\frac{2 N}{2 N-\alpha}} d x\right) \\
\leq & C^{\frac{2 N}{2 N-\alpha}} 2^{\frac{\alpha}{2 N-\alpha}}\left(\left\|\left|u_{n}\right|^{\frac{2 N}{2 N-\alpha}}\right\|_{L^{2}\left(\mathbb{R}^{N}\right)}\left\||\varphi|^{\frac{2 N}{2 N-\alpha}}\right\|_{L^{2}\left(\mathbb{R}^{N}\right)}\right. \\
& \left.+\left\|\left|u_{n}\right|^{(p-1) \frac{2 N}{2 N-\alpha}}\right\|_{L^{\frac{p}{p-1}}\left(\mathbb{R}^{N}\right)}\left\||\varphi|^{\frac{2 N}{2 N-\alpha}}\right\|_{L^{p}\left(\mathbb{R}^{N}\right)}\right) \\
\leq & C,
\end{aligned}
$$

thanks to $2<\frac{4 N}{2 N-\alpha}<2_{s}^{*}$ and $2<\frac{2 p N}{2 N-\alpha}<2_{s}^{*}$, where $C>0$ denotes various constants.

Clearly, $f\left(\left|u_{n}\right|^{2}\right) u_{n} \bar{\varphi} \rightarrow f\left(|u|^{2}\right) u \bar{\varphi}$ a.e. in $\mathbb{R}^{N}$. Hence, up to a subsequence, $\operatorname{Re}\left\{f\left(\left|u_{n}\right|^{2}\right) u_{n} \bar{\varphi}\right\}$ weakly converges to $\operatorname{Re}\left\{f\left(|u|^{2}\right) u \bar{\varphi}\right\}$ in $L^{\frac{2 N}{2 N-\alpha}}\left(\mathbb{R}^{N}\right)$. This together with (3.2) yields that

$$
\lim _{n \rightarrow \infty} \operatorname{Re} \int_{\mathbb{R}^{N}}\left(\mathcal{K}_{\alpha} * F\left(\left|u_{n}\right|^{2}\right)\right) f\left(\left|u_{n}\right|^{2}\right) u_{n} \bar{\varphi} d x=\lim _{n \rightarrow \infty} \operatorname{Re} \int_{\mathbb{R}^{N}}\left(\mathcal{K}_{\alpha} * F\left(|u|^{2}\right)\right) f\left(|u|^{2}\right) u \bar{\varphi} d x
$$

for each $\varphi \in E$.

We claim that as $n \rightarrow \infty$

$$
\int_{\mathbb{R}^{N}}\left|u_{n}\right|^{2_{s}^{*}} d x \rightarrow \int_{\mathbb{R}^{N}}|u|^{2_{s}^{*}} d x
$$

In order to prove this claim, we invoke Prokhorov's Theorem (see Theorem 8.6.2 in [9]) to conclude that there exist $\mu, \nu \in \mathcal{M}\left(\mathbb{R}^{N}\right)$ such that

$$
\begin{gathered}
\left|(-\Delta)^{\frac{s}{2}} u_{n}\right|^{2} \rightarrow \mu \quad\left(\text { weak }^{*} \text {-sense of measures }\right), \\
\left|u_{n}\right|^{2_{s}^{*}} \rightarrow \nu \quad\left(\text { weak }^{*} \text {-sense of measures }\right),
\end{gathered}
$$

where $\mu$ and $\nu$ are a nonnegative bounded measures on $\mathbb{R}^{N}$. It follows by Lemma 3.1 that either $u_{n} \rightarrow u$ in $L_{l o c}^{2_{s}^{*}}\left(\mathbb{R}^{N}\right)$ or $\nu=|u|^{2_{s}^{*}}+\sum_{j \in I} \delta_{x_{j}} \nu_{j}$, as $n \rightarrow \infty$, where $I$ is a countable set, $\left\{\nu_{j}\right\}_{j} \subset[0, \infty),\left\{x_{j}\right\}_{j} \subset \mathbb{R}^{N}$.

Take $\phi \in C_{0}^{\infty}\left(\mathbb{R}^{N}\right)$ such that $0 \leq \phi \leq 1 ; \phi \equiv 1$ in $B\left(x_{j}, \rho\right), \phi(x)=0$ in $\mathbb{R}^{N} \backslash B\left(x_{j}, 2 \rho\right)$. For any $\rho>0$, define $\phi_{\rho}=\phi\left(\frac{x-x_{j}}{\rho}\right)$, where $j \in I$. It follows that

$$
\begin{aligned}
& \iint_{\mathbb{R}^{2 N}} \frac{\left|u_{n}(x) \phi_{\rho}(x)-u_{n}(y) \phi_{\rho}(y)\right|^{2}}{|x-y|^{N+2 s}} d x d y \\
& \quad \leq 2 \iint_{\mathbb{R}^{2 N}} \frac{\left|u_{n}(x)-u_{n}(y)\right|^{2} \phi_{\rho}^{2}(y)}{|x-y|^{N+2 s}} d x d y+2 \iint_{\mathbb{R}^{2 N}} \frac{\left|\phi_{\rho}(x)-\phi_{\rho}(y)\right|^{2}\left|u_{n}(x)\right|^{2}}{|x-y|^{N+2 s}} d x d y \\
& \leq 2 \iint_{\mathbb{R}^{2 N}} \frac{\left|u_{n}(x)-u_{n}(y)\right|^{2}}{|x-y|^{N+2 s}} d x d y+2 \iint_{\mathbb{R}^{2 N}} \frac{\left|\phi_{\rho}(x)-\phi_{\rho}(y)\right|^{2}\left|u_{n}(x)\right|^{2}}{|x-y|^{N+2 s}} d x d y .
\end{aligned}
$$

Similar to the proof of Lemma 3.4 in [42], we can show that

$$
\iint_{\mathbb{R}^{2 N}} \frac{\left|\phi_{\rho}(x)-\phi_{\rho}(y)\right|^{2}\left|u_{n}(x)\right|^{2}}{|x-y|^{N+2 s}} d x d y \leq C \rho^{-2 s} \int_{B\left(x_{i}, K \rho\right)}\left|u_{n}(x)\right|^{2} d x+C K^{-N}
$$


where $K>4$. Since $\left\{u_{n}\right\}_{n}$ is bounded in $E$, it follows from (3.5) and (3.6) that $\left\{u_{n} \phi_{\rho}\right\}_{n}$ is bounded in $E$. Then $\left\langle J_{\varepsilon}^{\prime}\left(u_{n}\right), u_{n} \phi_{\rho}\right\rangle \rightarrow 0$, which implies

$$
\begin{aligned}
& M\left(\left[u_{n}\right]_{s, A}^{2}\right) \iint_{\mathbb{R}^{2 N}} \frac{\left|u_{n}(x)-e^{i(x-y) \cdot A\left(\frac{x+y}{2}\right)} u_{n}(y)\right|^{2} \phi_{\rho}(y)}{|x-y|^{N+2 s}} d x d y+\varepsilon^{-2 s} \int_{\mathbb{R}^{N}} V(x)\left|u_{n}\right|^{2} \phi_{\rho}(x) d x \\
& =-\operatorname{Re}\left\{M\left(\left[u_{n}\right]_{s, A}^{2}\right) \iint_{\mathbb{R}^{2 N}} \frac{\left(u_{n}(x)-e^{i(x-y) \cdot A\left(\frac{x+y}{2}\right)} u_{n}(y)\right) \overline{u_{n}(x)\left(\phi_{\rho}(x)-\phi_{\rho}(y)\right)}}{|x-y|^{N+2 s}} d x d y\right\} \\
& \left.+\varepsilon^{-2 s} \int_{\mathbb{R}^{N}}\left|u_{n}\right|^{2_{s}^{*}} \phi_{\rho} d x+\varepsilon^{-2 s} \int_{\mathbb{R}^{N}} \mathcal{K}_{\alpha} * F\left(\left|u_{n}\right|^{2}\right)\right) f\left(\left|u_{n}\right|^{2}\right)\left|u_{n}\right|^{2} \phi_{\rho} d x+o_{n}(1) .
\end{aligned}
$$

Note that by $\left(M_{2}\right)$ and diamagnetic inequality, the following holds

$$
\begin{aligned}
& M\left(\left[u_{n}\right]_{s, A}^{2}\right) \iint_{\mathbb{R}^{2 N}} \frac{\left|u_{n}(x)-e^{i(x-y) \cdot A\left(\frac{x+y}{2}\right)} u_{n}(y)\right|^{2} \phi_{\rho}(y)}{|x-y|^{N+2 s}} d x d y \\
& \geq m_{1}\left(\iint_{\mathbb{R}^{2 N}} \frac{\left|u_{n}(x)-e^{i(x-y) \cdot A\left(\frac{x+y}{2}\right)} u_{n}(y)\right|^{2} \phi_{\rho}(y)}{|x-y|^{N+2 s}} d x d y\right)^{2 \sigma} \\
& \geq m_{1}\left(\iint_{\mathbb{R}^{2 N}} \frac{\left.|| u_{n}(x)|-| u_{n}(y)\right|^{2} \phi_{\rho}(y)}{|x-y|^{N+2 s}} d x d y\right)^{2 \sigma} .
\end{aligned}
$$

It is easy to verify that

$$
\iint_{\mathbb{R}^{2 N}} \frac{\left\|u_{n}(x)|-| u_{n}(y)\right\|^{2} \phi_{\rho}(y)}{|x-y|^{N+2 s}} d x d y \rightarrow \int_{\mathbb{R}^{N}} \phi_{\rho} d \mu
$$

as $n \rightarrow \infty$ and

$$
\int_{\mathbb{R}^{N}} \phi_{\rho} d \mu \rightarrow \mu\left(\left\{x_{i}\right\}\right)
$$

as $\rho \rightarrow 0$. Note that the Hölder inequality implies

$$
\begin{aligned}
& \left|\operatorname{Re}\left\{M\left(\left[u_{n}\right]_{s, A}^{2}\right) \iint_{\mathbb{R}^{2 N}} \frac{\left(u_{n}(x)-e^{i(x-y) \cdot A\left(\frac{x+y}{2}\right)} u_{n}(y)\right) \overline{u_{n}(x)\left(\phi_{\rho}(x)-\phi_{\rho}(y)\right)}}{|x-y|^{N+2 s}} d x d y\right\}\right| \\
& \quad \leq C \iint_{\mathbb{R}^{2 N}} \frac{\left|u_{n}(x)-e^{i(x-y) \cdot A\left(\frac{x+y}{2}\right)} u_{n}(y)\right| \cdot\left|\phi_{\rho}(x)-\phi_{\rho}(y)\right| \cdot\left|u_{n}(x)\right|}{|x-y|^{N+2 s}} d x d y \\
& \quad \leq C\left(\iint_{\mathbb{R}^{2 N}} \frac{\left|u_{n}(x)\right|^{2}\left|\phi_{\rho}(x)-\phi_{\rho}(y)\right|^{2}}{|x-y|^{N+2 s}} d x d y\right)^{1 / 2} .
\end{aligned}
$$

Similar to the proof of Lemma 3.4 in [42], we can show that

$$
\lim _{\rho \rightarrow 0} \lim _{n \rightarrow \infty} \iint_{\mathbb{R}^{2 N}} \frac{\left|u_{n}(x)\right|^{2}\left|\phi_{\rho}(x)-\phi_{\rho}(y)\right|^{2}}{|x-y|^{N+2 s}} d x d y=0
$$

It follows from

$$
\left.\left.\lim _{n \rightarrow \infty} \int_{\mathbb{R}^{N}} \mathcal{K}_{\alpha} * F\left(\left|u_{n}\right|^{2}\right)\right) f\left(\left|u_{n}\right|^{2}\right)\left|u_{n}\right|^{2} \phi_{\rho} d x=\int_{\mathbb{R}^{N}} \mathcal{K}_{\alpha} * F\left(|u|^{2}\right)\right) f\left(|u|^{2}\right)|u|^{2} \phi_{\rho} d x
$$

and

$$
\left.\lim _{\rho \rightarrow 0} \int_{\mathbb{R}^{N}} \mathcal{K}_{\alpha} * F\left(|u|^{2}\right)\right) f\left(|u|^{2}\right)|u|^{2} \phi_{\rho} d x=0
$$


that

$$
\left.\lim _{\rho \rightarrow 0} \lim _{n \rightarrow \infty} \int_{\mathbb{R}^{N}} \mathcal{K}_{\alpha} * F\left(\left|u_{n}\right|^{2}\right)\right) f\left(\left|u_{n}\right|^{2}\right)\left|u_{n}\right|^{2} \phi_{\rho} d x=0 .
$$

Since $\phi_{\rho}$ has compact support, letting $n \rightarrow \infty$ in (3.7), we can deduce from (3.8)-(3.9) and the diamagnetic inequality that

$$
m_{1}\left(\mu\left(\left\{x_{j}\right\}\right)\right)^{2 \sigma} \leq \varepsilon^{-2 s} \nu_{j} .
$$

Combining this fact with Lemma 3.1, we obtain

$$
\nu_{j} \geq m_{1} \varepsilon^{2 s} S^{2 \sigma} \nu_{j}^{\frac{4 \sigma}{2 *}}
$$

This result implies that

$$
\text { (I) } \quad \nu_{j}=0 \quad \text { or } \quad \text { (II) } \quad \nu_{j} \geq\left(m_{1} S^{2 \sigma}\right)^{\frac{2_{s}^{*}}{22_{s}^{*}-4 \sigma}} \varepsilon^{\frac{2 s 2_{s}^{*}}{2-4 \sigma}} \text {. }
$$

To obtain the possible concentration of mass at infinity, we similarly define a cut off function $\phi_{R} \in C_{0}^{\infty}\left(\mathbb{R}^{N}\right)$ such that $\phi_{R}(x)=0$ on $|x|<R$ and $\phi_{R}(x)=1$ on $|x|>R+1$. We can verify that $\left\{u_{n} \phi_{R}\right\}_{n}$ is bounded in $E$, hence $\left\langle J_{\varepsilon}^{\prime}\left(u_{n}\right), u_{n} \phi_{R}\right\rangle \rightarrow 0$, as $n \rightarrow \infty$, which implies

$$
\begin{aligned}
& M\left(\left[u_{n}\right]_{s, A}^{2}\right) \iint_{\mathbb{R}^{2 N}} \frac{\left|u_{n}(x)-e^{i(x-y) \cdot A\left(\frac{x+y}{2}\right)} u_{n}(y)\right|^{2} \phi_{R}(y)}{|x-y|^{N+2 s}} d x d y+\varepsilon^{-2 s} \int_{\mathbb{R}^{N}} V(x)\left|u_{n}\right|^{2} \phi_{R}(x) d x \\
& =-\operatorname{Re}\left\{M\left(\left[u_{n}\right]_{s, A}^{2}\right) \iint_{\mathbb{R}^{2 N}} \frac{\left(u_{n}(x)-e^{i(x-y) \cdot A\left(\frac{x+y}{2}\right)} u_{n}(y)\right) \overline{u_{n}(x)\left(\phi_{R}(x)-\phi_{R}(y)\right)}}{|x-y|^{N+2 s}} d x d y\right\} \\
& \left.+\varepsilon^{-2 s} \int_{\mathbb{R}^{N}}\left|u_{n}\right|^{2_{s}^{*}} \phi_{R} d x+\varepsilon^{-2 s} \int_{\mathbb{R}^{N}} \mathcal{K}_{\alpha} * F\left(\left|u_{n}\right|^{2}\right)\right) f\left(\left|u_{n}\right|^{2}\right)\left|u_{n}\right|^{2} \phi_{R}(x) d x+o_{n}(1) .
\end{aligned}
$$

It is easy to verify that

$$
\limsup _{R \rightarrow \infty} \limsup _{n \rightarrow \infty} \iint_{\mathbb{R}^{2 N}} \frac{|| u_{n}(x)|-| u_{n}(y)||^{2} \phi_{R}(y)}{|x-y|^{N+2 s}} d x d y=\mu_{\infty}
$$

and

$$
\begin{aligned}
& \left|\operatorname{Re}\left\{M\left(\left[u_{n}\right]_{s, A}^{2}\right) \iint_{\mathbb{R}^{2 N}} \frac{\left(u_{n}(x)-e^{i(x-y) \cdot A\left(\frac{x+y}{2}\right)} u_{n}(y)\right) \overline{u_{n}(x)\left(\phi_{R}(x)-\phi_{R}(y)\right)}}{|x-y|^{N+2 s}} d x d y\right\}\right| \\
& \leq C\left(\iint_{\mathbb{R}^{2 N}} \frac{\left|u_{n}(x)\right|^{2}\left|\phi_{R}(x)-\phi_{R}(y)\right|^{2}}{|x-y|^{N+2 s}} d x d y\right)^{1 / 2} .
\end{aligned}
$$

Note that

$$
\begin{aligned}
& \limsup _{R \rightarrow \infty} \limsup _{n \rightarrow \infty} \iint_{\mathbb{R}^{2 N}} \frac{\left|u_{n}(x)\right|^{2}\left|\phi_{R}(x)-\phi_{R}(y)\right|^{2}}{|x-y|^{N+2 s}} d x d y \\
& =\limsup _{R \rightarrow \infty} \limsup _{n \rightarrow \infty} \iint_{\mathbb{R}^{2 N}} \frac{\left|u_{n}(x)\right|^{2}\left|\left(1-\phi_{R}(x)\right)-\left(1-\phi_{R}(y)\right)\right|^{2}}{|x-y|^{N+2 s}} d x d y .
\end{aligned}
$$

Similar to the proof of Lemma 3.4 in [42], we can show that

$$
\limsup _{R \rightarrow \infty} \limsup _{n \rightarrow \infty} \iint_{\mathbb{R}^{2 N}} \frac{\left|u_{n}(x)\right|^{2}\left|\left(1-\phi_{R}(x)\right)-\left(1-\phi_{R}(y)\right)\right|^{2}}{|x-y|^{N+2 s}} d x d y=0 .
$$


It follows from the fact that $\left(M_{2}\right)$, Lemma 2.1 and Lemma 3.2 that

$$
\begin{aligned}
& \limsup _{R \rightarrow \infty} \limsup _{n \rightarrow \infty} M\left(\left[u_{n}\right]_{s, A}^{2}\right) \iint_{\mathbb{R}^{2 N}} \frac{\left|u_{n}(x)-e^{i(x-y) \cdot A\left(\frac{x+y}{2}\right)} u_{n}(y)\right|^{2} \phi_{R}(y)}{|x-y|^{N+2 s}} d x d y \\
& \geq \limsup _{R \rightarrow \infty} \limsup _{n \rightarrow \infty} m_{1}\left(\iint_{\mathbb{R}^{2 N}} \frac{\left|u_{n}(x)-e^{i(x-y) \cdot A_{\varepsilon}\left(\frac{x+y}{2}\right)} u_{n}(y)\right|^{2} \phi_{R}(y)}{|x-y|^{N+2 s}} d x d y\right)^{2 \sigma} \\
& \quad \geq \limsup _{R \rightarrow \infty} \limsup _{n \rightarrow \infty} m_{1}\left(\iint_{\mathbb{R}^{2 N}} \frac{\left.|| u_{n}(x)|-| u_{n}(y)\right|^{2} \phi_{R}(y)}{|x-y|^{N+2 s}} d x d y\right)^{2 \sigma}=m_{1} \mu_{\infty}^{2 \sigma} .
\end{aligned}
$$

It is easy to see that

$$
\left.\lim _{R \rightarrow \infty} \lim _{n \rightarrow \infty} \int_{\mathbb{R}^{N}} \mathcal{K}_{\alpha} * F\left(\left|u_{n}\right|^{2}\right)\right) f\left(\left|u_{n}\right|^{2}\right)\left|u_{n}\right|^{2} \phi_{R}(x) d x=0 .
$$

By Lemma 3.2 and letting $R \rightarrow \infty$ in (3.10), we obtain

$$
\nu_{\infty} \geq m_{1} \varepsilon^{2 s} S^{2 \sigma} \nu_{\infty}^{\frac{4 \sigma}{2 *}} .
$$

This result implies that

$$
\text { (III) } \nu_{\infty}=0 \quad \text { or } \quad(\text { IV }) \quad \nu_{\infty} \geq\left(m_{1} S^{2 \sigma}\right)^{\frac{2_{s}^{*}}{2_{s}^{*}-4 \sigma}} \varepsilon^{\frac{2 s 2_{s}^{*}}{2 *-4 \sigma}} .
$$

Next, we claim that $(I I)$ and $(I V)$ cannot occur. If the case $(I V)$ holds for some $j \in I$, then by Lemma 3.2 , $(M)$ and $(H)$, we have

$$
\begin{aligned}
c \geq & \lim _{n \rightarrow \infty}\left(J_{\varepsilon}\left(u_{n}\right)-\frac{1}{\mu}\left\langle J_{\varepsilon}^{\prime}\left(u_{n}\right), u_{n}\right\rangle\right) \\
\geq & \left(\frac{1}{2 \sigma}-\frac{1}{\mu}\right) M\left(\left[u_{n}\right]_{s, A}^{2}\right)\left[u_{n}\right]_{s, A}^{2}+\left(\frac{1}{2}-\frac{1}{\mu}\right) \varepsilon^{-2 s} \int_{\mathbb{R}^{N}} V(x)\left|u_{n}\right|^{2} d x \\
& +\left(\frac{1}{\mu}-\frac{1}{2_{s}^{*}}\right) \varepsilon^{-2 s} \int_{\mathbb{R}^{N}}\left|u_{n}\right|^{2_{s}^{*}} d x+\varepsilon^{-2 s} \int_{\mathbb{R}^{N}}\left(\mathcal{K}_{\alpha} * F\left(\left|u_{n}\right|^{2}\right)\right)\left(\frac{1}{\mu} f\left(\left|u_{n}\right|^{2}\right)\left|u_{n}\right|^{2}-\frac{1}{4} F\left(\left|u_{n}\right|^{2}\right)\right) d x \\
\geq & \left(\frac{1}{\mu}-\frac{1}{2_{s}^{*}}\right) \varepsilon^{-2 s} \int_{\mathbb{R}^{N}}\left|u_{n}\right|^{2_{s}^{*}} d x \geq\left(\frac{1}{\mu}-\frac{1}{2_{s}^{*}}\right) \varepsilon^{-2 s} \nu_{\infty} \\
\geq & \left(\frac{1}{\mu}-\frac{1}{2_{s}^{*}}\right)\left(m_{1} S^{\sigma}\right)^{\frac{2_{s}^{*}}{2 s_{s}^{*}}} \varepsilon^{\frac{4 s \sigma}{22_{s}^{*}-4 \sigma}}=\sigma_{0} \varepsilon^{\frac{8 s \sigma}{2 *-4 \sigma}},
\end{aligned}
$$

where $\sigma_{0}=\left(\frac{1}{\mu}-\frac{1}{2_{s}^{*}}\right)\left(m_{1} S^{\sigma}\right)^{\frac{2_{s}^{*}}{2_{s}^{*}-2 \sigma}}$, which is impossible.

Consequently, $\nu_{j}=0$ for all $j \in I$. Similarly, we can prove that $(I I)$ cannot occur for any $j$. Thus

$$
\int_{\mathbb{R}^{N}}\left|u_{n}\right|^{2_{s}^{*}} d x \rightarrow \int_{\mathbb{R}^{N}}|u|^{2_{s}^{*}} d x .
$$

The Brézis-Lieb Lemma implies that

$$
\lim _{n \rightarrow \infty} \int_{\mathbb{R}^{N}}\left|u_{n}-u\right|^{2_{s}^{*}} d x=0 .
$$

Therefore, we get

$$
u_{n} \rightarrow u \quad \text { in } \quad L^{2_{s}^{*}}\left(\mathbb{R}^{N}\right) \quad \text { as } \quad n \rightarrow \infty .
$$


By the weak lower semicontinuity of the norm, conditon $\left(m_{1}\right)$ and the Brézis-Lieb Lemma, we have

$$
\begin{aligned}
o(1)\left\|u_{n}\right\|_{\varepsilon}= & \left\langle J_{\varepsilon}^{\prime}\left(u_{n}\right), u_{n}\right\rangle=M\left(\left[u_{n}\right]_{s, A}^{2}\right)\left[u_{n}\right]_{s, A}^{2}+\varepsilon^{-2 s} \int_{\mathbb{R}^{N}} V(x)\left|u_{n}\right|^{2} d x \\
& -\varepsilon^{-2 s} \int_{\mathbb{R}^{N}}\left|u_{n}\right|^{2_{s}^{*}} d x-\varepsilon^{-2 s} \int_{\mathbb{R}^{N}} \mathcal{K}_{\alpha} * F\left(\left|u_{n}\right|^{2}\right) f\left(\left|u_{n}\right|^{2}\right)\left|u_{n}\right|^{2} d x \\
\geq & m_{1}\left(\left[u_{n}\right]_{s, A}^{2 \sigma}-[u]_{s, A}^{2 \sigma}\right)+\varepsilon^{-2 s} \int_{\mathbb{R}^{N}} V(x)\left(\left|u_{n}\right|^{2}-|u|^{2}\right) d x+M\left([u]_{s, A}^{2}\right)[u]_{s, A}^{2} \\
& +\varepsilon^{-2 s} \int_{\mathbb{R}^{N}} V(x)|u|^{2} d x-\varepsilon^{-2 s} \int_{\mathbb{R}^{N}}|u|^{2_{s}^{*}} d x-\varepsilon^{-2 s} \int_{\mathbb{R}^{N}} \mathcal{K}_{\alpha} * F\left(|u|^{2}\right) f\left(|u|^{2}\right)|u|^{2} d x \\
\geq & \min \left\{m_{1}, 1\right\} \min \left\{\left\|u_{n}-u\right\|_{\varepsilon}^{2 \sigma},\left\|u_{n}-u\right\|_{\varepsilon}^{2}\right\}+o(1)\|u\|_{\varepsilon} .
\end{aligned}
$$

Here we use the fact that $J_{\varepsilon}^{\prime}(u)=0$. Thanks to $2<2 \sigma$, we have proved that $\left\{u_{n}\right\}_{n}$ strongly converges to $u$ in $E$. Hence the proof is complete.

\section{Proofs of Main Theorems}

In this section, we shall prove our main results. We shall first establish Theorem 1.1.

Note that $J_{\varepsilon}(u)$ does not satisfy $(P S)_{c}$ condition for any $c>0$. Thus, in the sequel we shall find a special finite-dimensional subspace by which we construct sufficiently small minimax levels.

Recall that the assumption $(V)$ implies that there is $x_{0} \in \mathbb{R}^{N}$ such that $V\left(x_{0}\right)=\min _{x \in \mathbb{R}^{N}} V(x)=0$. Without loss of generality we can assume from now on that $x_{0}=0$.

Proposition 4.1. (see $\left[8\right.$, Theorem 3.2]) For any $q \in\left(2,2_{s}^{*}\right)$, we have

$$
\inf \left\{\iint_{\mathbb{R}^{2 N}} \frac{|\phi(x)-\phi(y)|^{2}}{|x-y|^{N+2 s}} d x d y: \phi \in C_{0}^{\infty}\left(\mathbb{R}^{N}\right),|\phi|_{q}=1\right\}=0 .
$$

By Proposition 4.1, one can choose $\phi_{\zeta} \in C_{0}^{\infty}\left(\mathbb{R}^{N}\right)$ with $\left|\phi_{\zeta}\right|_{q}=1$ and $\operatorname{supp} \phi_{\zeta} \subset B_{r_{\zeta}}(0)$ so that

$$
\iint_{\mathbb{R}^{2 N}} \frac{\left|\phi_{\zeta}(x)-\phi_{\zeta}(y)\right|^{2}}{|x-y|^{N+2 s}} d x d y \leq C \zeta^{\frac{2 N-(N-2 s) q}{q}}
$$

for any $1>\zeta>0$.

Set

$$
\psi_{\zeta}(x)=e^{i A(0) x} \phi_{\zeta}(x), \quad \psi_{\varepsilon, \zeta}(x)=\psi_{\zeta}\left(\varepsilon^{-\tau} x\right)
$$

and

$$
\tau:=\frac{2 s 2_{s}^{*}}{N\left(2_{s}^{*}-4 \sigma\right)}
$$

By $\left(f_{3}\right)$, for any $t>0$ we get

$$
\begin{aligned}
J_{\varepsilon}\left(t \psi_{\varepsilon, \zeta}\right) \leq & \frac{C_{0}}{2} t^{2 \sigma}\left(\iint_{\mathbb{R}^{2 N}} \frac{\left|\psi_{\varepsilon, \zeta}(x)-e^{i(x-y) \cdot A\left(\frac{x+y}{2}\right)} \psi_{\varepsilon, \zeta}(y)\right|^{2}}{|x-y|^{N+2 s}} d x d y\right)^{2 \sigma} \\
& +\frac{t^{2}}{2} \varepsilon^{-2 s} \int_{\mathbb{R}^{N}} V(x)\left|\psi_{\varepsilon, \zeta}\right|^{2} d x-t^{2_{s}^{*}} \frac{\varepsilon^{-2 s}}{2_{s}^{*}} \int_{\mathbb{R}^{N}}|u|^{2_{s}^{*}} d x \\
\leq & \varepsilon^{N \tau-2 s}\left[\frac{C_{0}}{2} t^{2 \sigma}\left(\iint_{\mathbb{R}^{2 N}} \frac{\left|\psi_{\zeta}(x)-e^{i\left(\varepsilon^{\tau} x-\varepsilon^{\tau} y\right) \cdot A\left(\frac{\varepsilon^{\tau} x+\varepsilon^{\tau} y}{2}\right)} \psi_{\zeta}(y)\right|^{2}}{|x-y|^{N+2 s}} d x d y\right)^{2 \sigma}\right. \\
& \left.+\frac{t^{2}}{2} \int_{\mathbb{R}^{N}} V\left(\varepsilon^{\tau} x\right)\left|\psi_{\zeta}\right|^{2} d x-\frac{t_{s}^{2_{s}^{*}}}{2_{s}^{*}} \int_{\mathbb{R}^{N}}\left|\psi_{\zeta}\right|^{2_{s}^{*}} d x\right] \\
= & \varepsilon^{\frac{8 s \sigma}{2 *-4 \sigma}} I_{\varepsilon}\left(t \psi_{\zeta}\right),
\end{aligned}
$$


where $I_{\varepsilon} \in C^{1}(E, \mathbb{R})$ is defined by

$$
\begin{aligned}
I_{\varepsilon}(u):= & \frac{C_{0}}{2}\left(\iint_{\mathbb{R}^{2 N}} \frac{\left|u(x)-e^{i\left(\varepsilon^{\tau} x-\varepsilon^{\tau} y\right) \cdot A\left(\frac{\varepsilon^{\tau} x+\varepsilon^{\tau} y}{2}\right)} u(y)\right|^{2}}{|x-y|^{N+2 s}} d x d y\right)^{2 \sigma} \\
& +\frac{1}{2} \int_{\mathbb{R}^{N}} V\left(\varepsilon^{\tau} x\right)|u|^{2} d x-\frac{1}{2_{s}^{*}} \int_{\mathbb{R}^{N}}|u|^{2_{s}^{*}} d x .
\end{aligned}
$$

Since $2_{s}^{*}>2 \sigma$, there exists a finite number $t_{0} \in[0,+\infty)$ such that

$$
\begin{aligned}
\max _{t \geq 0} I_{\varepsilon}\left(t \psi_{\zeta}\right)= & \frac{C_{0}}{2} t_{0}^{2 \sigma}\left(\iint_{\mathbb{R}^{2 N}} \frac{\left|\psi_{\zeta}(x)-e^{i\left(\varepsilon^{\tau} x-\varepsilon^{\tau} y\right) \cdot A\left(\frac{\varepsilon^{\tau} x+\varepsilon^{\tau} y}{2}\right)} \psi_{\zeta}(y)\right|^{2}}{|x-y|^{N+2 s}} d x d y\right)^{2 \sigma} \\
& +\frac{t_{0}^{2}}{2} \int_{\mathbb{R}^{N}} V\left(\varepsilon^{\tau} x\right)\left|\psi_{\zeta}\right|^{2} d x-\frac{t_{0}^{2_{s}^{*}}}{2_{s}^{*}} \int_{\mathbb{R}^{N}}\left|\psi_{\zeta}\right|^{2_{s}^{*}} d x \\
\leq & \frac{C_{0}}{2} t_{0}^{2 \sigma}\left(\iint_{\mathbb{R}^{2 N}} \frac{\left|\psi_{\zeta}(x)-e^{i\left(\varepsilon^{\tau} x-\varepsilon^{\tau} y\right) \cdot A\left(\frac{\varepsilon^{\tau} x+\varepsilon^{\tau} y}{2}\right)} \psi_{\zeta}(y)\right|^{2}}{|x-y|^{N+2 s}} d x d y\right)^{2 \sigma} \\
& +\frac{t_{0}^{2}}{2} \int_{\mathbb{R}^{N}} V\left(\varepsilon^{\tau} x\right)\left|\psi_{\zeta}\right|^{2} d x .
\end{aligned}
$$

Let $\psi_{\zeta}(x)=e^{i A(0) x} \phi_{\zeta}(x)$, where $\phi_{\zeta}(x)$ is as defined above. Then we have the following lemma.

Lemma 4.1. (see [8, Lemma 3.6]) (Norm estimate) For any $\zeta>0$ there exists $\varepsilon_{0}=\varepsilon_{0}(\zeta)>0$ such that

$$
\iint_{\mathbb{R}^{2 N}} \frac{\left|\psi_{\zeta}(x)-e^{i\left(\varepsilon^{\tau} x-\varepsilon^{\tau} y\right) \cdot A\left(\frac{\varepsilon^{\tau} x+\varepsilon^{\tau} y}{2}\right)} \psi_{\zeta}(y)\right|^{2}}{|x-y|^{N+2 s}} d x d y \leq C \zeta^{\frac{2 N-(N-2 s) q}{q}}+\frac{1}{1-s} \zeta^{2 s}+\frac{4}{s} \zeta^{2 s},
$$

for all $0<\varepsilon<\varepsilon_{0}$ and some constant $C>0$ depending only on $[\phi]_{s, 0}$.

On the one hand, since $V(0)=0$ and note that $\operatorname{supp} \phi_{\zeta} \subset B_{r_{\zeta}}(0)$, there is $\varepsilon^{*}>0$ such that

$$
V\left(\varepsilon^{\tau} x\right) \leq \frac{\zeta}{\left|\phi_{\zeta}\right|_{2}^{2}} \quad \text { for all }|x| \leq r_{\zeta} \text { and } 0<\varepsilon<\varepsilon^{*} .
$$

This implies that

$$
\max _{t \geq 0} I_{\varepsilon}\left(t \phi_{\delta}\right) \leq \frac{C_{0}}{2} t_{0}^{2 \sigma}\left(C \zeta^{\frac{2 N-(N-2 s) q}{q}}+\frac{1}{1-s} \zeta^{2 s}+\frac{4}{s} \zeta^{2 s}\right)^{2 \sigma}+\frac{t_{0}^{2}}{2} \zeta
$$

Therefore, for all $0<\varepsilon<\min \left\{\varepsilon_{0}, \varepsilon^{*}\right\}$, we have

$$
\max _{t \geq 0} J_{\varepsilon}\left(t \psi_{\lambda, \zeta}\right) \leq\left[\frac{C_{0}}{2} t_{0}^{2 \sigma}\left(C \zeta^{\frac{2 N-(N-2 s) q}{q}}+\frac{1}{1-s} \zeta^{2 s}+\frac{4}{s} \zeta^{2 s}\right)^{2 \sigma}+\frac{t_{0}^{2}}{2} \zeta\right] \varepsilon^{\frac{8 s \sigma}{2 *-4 \sigma}}
$$

Thus we have the following result.

Lemma 4.2. Under the assumptions of Lemma 2.2, for any $\kappa>0$ there exists $\mathcal{E}_{\kappa}>0$ such that for each $0<\varepsilon<\mathcal{E}_{\kappa}$, there is $\widehat{e}_{\varepsilon} \in E$ with $\left\|\widehat{e}_{\varepsilon}\right\|>\varrho_{\varepsilon}, J_{\varepsilon}\left(\widehat{e}_{\varepsilon}\right) \leq 0$ and

$$
\max _{t \in[0,1]} J_{\varepsilon}\left(t \widehat{e}_{\varepsilon}\right) \leq \kappa \varepsilon^{\frac{8 s \sigma}{2 s-4 \sigma}} .
$$

Proof. Choose $\zeta>0$ so small that

$$
\frac{C_{0}}{2} t_{0}^{2 \sigma}\left(C \zeta^{\frac{2 N-(N-2 s) q}{q}}+\frac{1}{1-s} \zeta^{2 s}+\frac{4}{s} \zeta^{2 s}\right)^{2 \sigma}+\frac{t_{0}^{2}}{2} \zeta \leq \kappa .
$$

Let $\psi_{\varepsilon, \zeta} \in E$ be the function defined by (4.2). Set $\mathcal{E}_{\kappa}=\min \left\{\varepsilon_{0}, \varepsilon^{*}\right\}$. Let $\widehat{t}_{\varepsilon}>0$ be such that $\widehat{t_{\varepsilon}}\left\|\psi_{\varepsilon, \zeta}\right\|_{\varepsilon}>\varrho_{\varepsilon}$ and $J_{\varepsilon}\left(t \psi_{\varepsilon, \zeta}\right) \leq 0$ for all $t \geq \widehat{t}_{\varepsilon}$. By (4.4), let $\widehat{e}_{\varepsilon}=\widehat{t}_{\varepsilon} \psi_{\varepsilon, \zeta}$ we know that the conclusion of Lemma 4.2 holds. 
Proof of Theorem 1.1. For any $0<\kappa<\sigma_{0}$, by Lemma 3.3, we choose $\mathcal{E}_{\kappa}>0$ and define for $0<\varepsilon<\mathcal{E}_{\kappa}$, the minimax value

$$
c_{\varepsilon}:=\inf _{\gamma \in \Gamma_{\varepsilon}} \max _{t \in[0,1]} J_{\varepsilon}\left(t \widehat{e}_{\varepsilon}\right),
$$

where

$$
\Gamma_{\varepsilon}:=\left\{\gamma \in C([0,1], E): \gamma(0)=0 \text { and } \gamma(1)=\widehat{e}_{\varepsilon}\right\} .
$$

By Lemma 2.2, we have $\alpha_{\varepsilon} \leq c_{\varepsilon} \leq \kappa \varepsilon^{\frac{8 s \sigma}{22_{s}^{*}-4 \sigma}}$. By virtue of Lemma 3.3, we know that $J_{\varepsilon}$ satisfies the $(P S)_{c_{\lambda}}$ condition, there is $u_{\varepsilon} \in E$ such that $J_{\varepsilon}^{\prime}\left(u_{\varepsilon}\right)=0$ and $J_{\varepsilon}\left(u_{\varepsilon}\right)=c_{\varepsilon}$, Then $u_{\varepsilon}$ is a nontrivial mountain pass solution of problem (2.2).

Since $u_{\varepsilon}$ is a critical point of $J_{\varepsilon}$, by $(M)$ and $(H)$, we have for $\tau \in\left[2 \sigma, 2_{s}^{*}\right]$

$$
\begin{aligned}
\kappa \varepsilon^{\frac{8 s \sigma}{2 *-4 \sigma} \geq} & J_{\varepsilon}\left(u_{\varepsilon}\right)=J_{\varepsilon}\left(u_{\varepsilon}\right)-\frac{1}{\tau}\left\langle J_{\varepsilon}^{\prime}\left(u_{\varepsilon}\right), u_{\varepsilon}\right\rangle \\
= & \frac{1}{2} \widetilde{M}\left(\left[u_{\varepsilon}\right]_{s, A_{\varepsilon}}^{2}\right)-\frac{1}{\tau} M\left(\left[u_{\varepsilon}\right]_{s, A_{\varepsilon}}^{2}\right)\left[u_{\varepsilon}\right]_{s, A_{\varepsilon}}^{2}+\left(\frac{1}{2}-\frac{1}{\tau}\right) \varepsilon^{-2 s} \int_{\mathbb{R}^{N}} V(x)\left|u_{\varepsilon}\right|^{2} d x \\
& +\left(\frac{1}{\tau}-\frac{1}{2_{s}^{*}}\right) \varepsilon^{-2 s} \int_{\mathbb{R}^{N}}\left|u_{\varepsilon}\right|^{2_{s}^{*}} d x+\varepsilon^{-2 s} \int_{\mathbb{R}^{N}}\left(\mathcal{K}_{\alpha} * F\left(\left|u_{\varepsilon}\right|^{2}\right)\right)\left(\frac{1}{\tau} f\left(\left|u_{\varepsilon}\right|^{2}\right)\left|u_{\varepsilon}\right|^{2}-\frac{1}{4} F\left(\left|u_{\varepsilon}\right|^{2}\right)\right) d x \\
\geq & \left(\frac{1}{2 \sigma}-\frac{1}{\tau}\right) m_{1}\left[u_{\varepsilon}\right]_{s, A_{\varepsilon}}^{2 \sigma}+\left(\frac{1}{2}-\frac{1}{\tau}\right) \varepsilon^{-2 s} \int_{\mathbb{R}^{N}} V(x)\left|u_{\varepsilon}\right|^{2} d x \\
& +\left(\frac{1}{\tau}-\frac{1}{2_{s}^{*}}\right) \varepsilon^{-2 s} \int_{\mathbb{R}^{N}}\left|u_{\varepsilon}\right|^{2_{s}^{*}} d x+\left(\frac{\mu}{\tau}-\frac{1}{4}\right) \varepsilon^{-2 s} \iint_{\mathbb{R}^{2 N}} \frac{F\left(\left|u_{\varepsilon}(x)\right|^{2}\right) F\left(\left|u_{\varepsilon}(y)\right|^{2}\right)}{|x-y|^{\alpha}} d x d y .
\end{aligned}
$$

Taking $\tau=2 / \sigma$, we obtain the estimate (1.7) and taking $\tau=\mu$ we obtain the estimate (1.8). This completes the proof of Theorem 1.1.

Next, we shall establish Theorem 1.2. Again, we shall first need to prove a lemma.

For any $m^{*} \in \mathbb{N}$, one can choose $m^{*}$ functions $\phi_{\zeta}^{i} \in C_{0}^{\infty}\left(\mathbb{R}^{N}\right)$ such that $\operatorname{supp} \phi_{\zeta}^{i} \cap \operatorname{supp} \phi_{\zeta}^{k}=\emptyset, i \neq k,\left|\phi_{\zeta}^{i}\right|_{s}=1$ and

$$
\iint_{\mathbb{R}^{2 N}} \frac{\left|\phi_{\zeta}^{i}(x)-\phi_{\zeta}^{i}(y)\right|^{2}}{|x-y|^{N+2 s}} d x d y \leq C \zeta^{\frac{2 N-(N-2 s) q}{q}} .
$$

Let $r_{\zeta}^{m^{*}}>0$ be such that $\operatorname{supp} \phi_{\zeta}^{i} \subset B_{r_{\zeta}}^{i}(0)$ for $i=1,2, \cdots, m^{*}$. Set

$$
\psi_{\zeta}^{i}(x)=e^{i A(0) x} \phi_{\zeta}^{i}(x)
$$

and

$$
\psi_{\varepsilon, \zeta}^{i}(x)=\psi_{\zeta}^{i}\left(\varepsilon^{-1} x\right)
$$

Denote

$$
\mathcal{H}_{\varepsilon \zeta}^{m^{*}}=\operatorname{span}\left\{\psi_{\varepsilon, \zeta}^{1}, \psi_{\varepsilon, \zeta}^{2}, \cdots, \psi_{\varepsilon, \zeta}^{m^{*}}\right\}
$$

Observe that for each $u=\sum_{i=1}^{m^{*}} c_{i} \psi_{\varepsilon, \zeta}^{i} \in \mathcal{H}_{\varepsilon \zeta}^{m^{*}}$, we have

$$
[u]_{s, A_{\varepsilon}}^{2} \leq C \sum_{i=1}^{m^{*}}\left|c_{i}\right|^{2}\left[\psi_{\varepsilon, \zeta}^{i}\right]_{s, A_{\varepsilon}}^{2}
$$

for some constant $C>0$. Therefore

$$
J_{\varepsilon}(u) \leq C \sum_{i=1}^{m^{*}} J_{\varepsilon}\left(c_{i} \psi_{\varepsilon, \zeta}^{i}\right)
$$

for some constant $C>0$. Based on a similar argument as before, we see that

$$
J_{\varepsilon}\left(c_{i} \psi_{\varepsilon, \zeta}^{i}\right) \leq \varepsilon^{N-2 s} \Psi\left(\left|c_{i}\right| \psi_{\zeta}^{i}\right) .
$$


As before, we can obtain the following estimate:

$$
\max _{u \in \mathcal{H}_{\varepsilon \delta}^{m *}} J_{\varepsilon}(u) \leq C m^{*}\left[\frac{C_{0}}{2} t_{0}^{2 \sigma}\left(C \zeta^{\frac{2 N-(N-2 s) q}{q}}+\frac{1}{1-s} \zeta^{2 s}+\frac{4}{s} \zeta^{2 s}\right)^{2 \sigma}+\frac{t_{0}^{2}}{2} \zeta\right] \varepsilon^{\frac{8 s \sigma}{2 *-4 \sigma}}
$$

for all small enough $\zeta$ and some constant $C>0$. From the estimate (4.9) we have the following:

Lemma 4.3. Under the assumptions of Lemma 2.2, for any $m^{*} \in \mathbb{N}$ and $\kappa>0$ there exists $\mathcal{E}_{m^{*} \kappa}>0$ such that for each $0<\varepsilon<\mathcal{E}_{m^{*} \kappa}$, there exists an $m^{*}$-dimensional subspace $\mathcal{F}_{\lambda m^{*}}$ satisfying

$$
\max _{u \in \mathcal{F}_{\lambda m^{*}}} J_{\varepsilon}(u) \leq \kappa \varepsilon^{\frac{8 s \sigma}{22_{s}^{2}-4 \sigma}}
$$

Proof. Choose $\zeta>0$ so small that

$$
C m^{*}\left[\frac{C_{0}}{2} t_{0}^{2 \sigma}\left(C \zeta^{\frac{2 N-(N-2 s) q}{q}}+\frac{1}{1-s} \zeta^{2 s}+\frac{4}{s} \zeta^{2 s}\right)^{2 \sigma}+\frac{t_{0}^{2}}{2} \zeta\right] \leq \kappa .
$$

Set $\mathcal{F}_{\varepsilon m^{*}}=\mathcal{H}_{\varepsilon \zeta}^{m^{*}}=\operatorname{span}\left\{\psi_{\varepsilon, \zeta}^{1}, \psi_{\varepsilon, \zeta}^{2}, \cdots, \psi_{\varepsilon, \zeta}^{m^{*}}\right\}$. Now the conclusion of Lemma 4.3 follows from (4.9).

Proof of Theorem 1.2. Denote the set of all symmetric (in the sense that $-Z=Z$ ) and closed subsets of $E$ by $\Sigma$, for each $Z \in \Sigma$. Let $\operatorname{gen}(Z)$ be the Krasnoselski genus and

$$
j(Z):=\min _{\eta \in \Phi_{m^{*}}} \operatorname{gen}\left(\eta(Z) \cap \partial B_{\varrho_{\varepsilon}}\right),
$$

where $\Phi_{m^{*}}$ is the set of all odd homeomorphisms $\eta \in C(E, E)$ and $\varrho_{\varepsilon}$ is the number from Lemma 2.2. Then $j$ is a version of Benci's pseudoindex [6]. Let

$$
c_{\varepsilon i}:=\inf _{j(Z) \geq i} \sup _{u \in Z} J_{\varepsilon}(u), \quad 1 \leq i \leq m^{*} .
$$

Since $J_{\varepsilon}(u) \geq \alpha_{\varepsilon}$ for all $u \in \partial B_{\varrho_{\varepsilon}}^{+}$and since $j\left(\mathcal{F}_{\varepsilon m^{*}}\right)=\operatorname{dim} \mathcal{F}_{\varepsilon m^{*}}=m^{*}$, we obtain

$$
\alpha_{\varepsilon} \leq c_{\varepsilon 1} \leq \cdots \leq c_{\varepsilon m^{*}} \leq \sup _{u \in H_{\varepsilon m^{*}}} J_{\varepsilon}(u) \leq \kappa \varepsilon^{\frac{8 s \sigma}{2 s-4 \sigma}}
$$

It follows from Lemma 3.3 that $J_{\varepsilon}$ satisfies the $(P S)_{c_{\varepsilon}}$ condition at all levels $c<\sigma_{0} \varepsilon^{N-2 s}$. By the usual critical point theory, all $c_{\varepsilon i}$ are critical levels and $J_{\varepsilon}$ has at least $m^{*}$ pairs of nontrivial critical points satisfying

$$
\alpha_{\varepsilon} \leq J_{\varepsilon}\left(u_{\varepsilon}\right) \leq \kappa \varepsilon^{\frac{8 s \sigma}{2 *-4 \sigma}} .
$$

Hence, problem (2.2) has at least $m^{*}$ pairs of solutions. Finally, as in the proof of Theorem 1.1, we see that these solutions satisfy the estimates (1.7) and (1.8).

\section{Acknowledgements}

The first author was supported by the Research Foundation during the 13th Five-Year Plan Period of Department of Education of Jilin Province (JJKH20170648KJ), Natural Science Foundation of Changchun Normal University (No. 2017-09). The second author was supported by the Slovenian Research Agency (No. P1-0292, N1-0114, N1-0083, N1-0064, and J1-8131). The third author was supported by the National Natural Science Foundation of China (No. 11871199) and Heilongjiang Province Postdoctoral Startup Foundation (LBH-Q18109). 


\section{References}

[1] C.O. Alves, J.M. do Ó, O.H. Miyagaki, Concentration phenomena for fractional elliptic equations involving exponential critical growth, Adv. Nonlinear Stud. 16 (2016) 843-861.

[2] V. Ambrosio, Concentration phenomena for a fractional Choquard equation with magnetic field, Dynam. Part. Differ. Eq. 16 (2019) 125-149.

[3] D. Applebaum, Lévy processes-from probalility to finance and quantum groups, Notices Amer. Math. Soc. 51 (2004) 1336-1347.

[4] G. Arioli, A. Szulkin, A semilinear Schrödinger equation in the presence of a magnetic field, Arch. Rational Mech. Anal. 170 (2003) 277-295.

[5] G. Autuori, A. Fiscella, P. Pucci, Stationary Kirchhoff problems involving a fractional operator and a critical nonlinearity, Nonlinear Anal. 125 (2015) 699-714.

[6] V. Benci, On critical point theory of indefinite functionals in the presence of symmetries, Trans. Amer. Math. Soc. 274 (1982) 533-572.

[7] Z. Binlin, G. Molica Bisci, R. Servadei, Superlinear nonlocal fractional problems with infinitely many solutions, Nonlinearity 28 (2015) 2247-2264.

[8] Z. Binlin, M. Squassina, X. Zhang, Fractional NLS equations with magnetic field, critical frequency and critical growth, Manuscripta Math. 291 (2018) 1553-1546.

[9] V.I. Bogachev, Measure Theory, vol. II, xiv+575 pp, Springer-Verlag, Berlin, 2007.

[10] S. Cingolani, S. Secchi, Semiclassical limit for nonlinear Schrödinger equations with electromagnetic fileds, J. Math. Anal. Appl. 275 (2002) 108-130.

[11] P. d'Avenia, M. Squassina, Ground states for fractional magnetic operators, ESAIM: Control Optim. Calc. Var. 24 (2018) 1-24.

[12] J. Di Cosmo, J. Van Schaftingen, Semiclassical stationary states for nonlinear Schrödinger equations under a strong extenal magnetic field, J. Differential Equations 259 (2015) 596-627.

[13] E. Di Nezza, G. Palatucci, E. Valdinoci, Hitchhiker's guide to the fractional Sobolev spaces, Bull. Sci. Math. 136 (2012) 521-573.

[14] Y. Ding, F. Lin, Solutions of perturbed Schrödinger equations with critical nonlinearity, Calc. Var. Partial Differential Equations 30 (2007) 231-249.

[15] A. Fiscella, E. Valdinoci, A critical Kirchhoff type problem involving a nonlocal operator, Nonlinear Anal. 94 (2014) 156-170.

[16] T. Ichinose, Essential selfadjointness of the Weyl quantized relativistic Hamiltonian, Ann. Inst. H. Poincare Phys. Theor. 51 (1989) 265-297.

[17] T. Ichinose, H. Tamura, Imaginary-time path integral for a relativistic spinless particle in an electromagnetic field, Comm. Math. Phys. 105 (1986) 239-257.

[18] V. Iftimie, M. Măntoiu, R. Purice, Magnetic pseudodifferential operators, Publ. Res. Inst. Math. Sci. 43 (2007) 585-623.

[19] K. Kurata, Existence and semi-classical limit of the least energy solution to a nonlinear Schrödinger equation with electromagnetic fileds, Nonlinear Anal. 41 (2000) 763-778. 
[20] S. Liang, D. Repovš, B. Zhang, On the fractional Schrödinger-Kirchhoff equations with electromagnetic fields and critical nonlinearity, Comput. Math. Appl. 75 (2018) 1778-1794.

[21] S. Liang, S. Shi, Soliton solutions to Kirchhoff type problems involving the critical growth in $\mathbb{R}^{N}$, Nonlinear Anal. 81 (2013) 31-41.

[22] S. Liang, J. Zhang, Existence of solutions for Kirchhoff type problems with critical nonlinearity in $\mathbb{R}^{3}$, Nonlinear Anal. Real World Appl. 17 (2014) 126-136.

[23] D. Lü, Existence and concentration behavior of ground state solutions for magnetic nonlinear Choquard equations, Commun. Pure Appl. Anal. 15 (2016) 1781-1795.

[24] X. Mingqi, P. Pucci, M. Squassina, B. Zhang, Nonlocal Schrödinger-Kirchhoff equations with external magnetic field, Discrete Contin. Dyn. Syst. 37 (2017) 503-521.

[25] G. Molica Bisci, V. Rădulescu, R. Servadei, Variational methods for nonlocal fractional equations, Encyclopedia of Mathematics and its Applications, 162, Cambridge University Press, Cambridge, 2016.

[26] G. Molica Bisci, V. Rădulescu, Ground state solutions of scalar field fractional for Schrödinger equations, Calc. Var. Partial Differential Equations 54 (2015) 2985-3008.

[27] G. Molica Bisci, D. Repovš, On doubly nonlocal fractional elliptic equations, Rend. Lincei Mat. Appl. 26 (2015) 161-176.

[28] G. Palatucci, A. Pisante, Improved Sobolev embeddings, profile decomposition, and concentrationcompactness for fractional Sobolev spaces. Calc. Var. Partial Differential Equations 50 (2014) 799-829.

[29] P. Pucci, M. Xiang, B. Zhang, Multiple solutions for nonhomogeneous Schrödinger-Kirchhoff type equations involving the fractional $p$-Laplacian in $\mathbb{R}^{N}$, Calc. Var. Partial Differetial Equations 54 (2015) 2785-2806.

[30] P. Pucci, M. Xiang, B. Zhang, Existence and multiplicity of entire solutions for fractional $p$-Kirchhoff equations, Adv. Nonlinear Anal. 5 (2016) 27-55.

[31] P. Pucci, M. Xiang, B. Zhang, Existence results for Schrödinger-Choquard-Kirchhoff equations involving the fractional p-Laplacian, Adv. Calc. Var. 12 (2019) 253-275.

[32] P.H. Rabinowitz, Minimax methods in critical point theory with applications to differential equations, in: CBME Regional Conference Series in Mathematics, vol. 65, American Mathematical Society, Providence, RI, 1986.

[33] M. Squassina, Soliton dynamics for the nonlinear Schrödinger equation with magnetic field, Manuscripta Math. 130 (2009) 461-494.

[34] M. Squassina, B. Volzone, Bourgain-Brézis-Mironescu formula for magnetic operators, C. R. Math. 354 (2016) 825-831.

[35] F. Wang, M. Xiang, Multiplicity of solutions to a nonlocal Choquard equation involving fractional magnetic operators and critical exponent, Electron. J. Differ. Eq. 2016 (2016) 1-11.

[36] M. Willem, Minimax Theorems, Birkhäser, Boston, 1996.

[37] M. Xiang, B. Zhang, V. Rădulescu, Multiplicity of solutions for a class of quasilinear Kirchhoff system involving the fractional $p$-Laplacian, Nonlinearity 29 (2016) 3186-3205.

[38] M. Xiang, B. Zhang, V. Rădulescu, Superlinear Schrödinger-Kirchhoff type problems involving the fractional p-Laplacian and critical exponent, Adv. Nonlinear Anal. 9 (2020) 690-709.

[39] M. Xiang, B. Zhang, X. Zhang, A nonhomogeneous fractional $p$-Kirchhoff type problem involving critical exponent in $\mathbb{R}^{N}$, Adv. Nonlinear Stud. 17 (2017) 611-640. 
[40] J. Yang, F. Wu, Doubly critical problems involving fractional Laplacians in $\mathbb{R}^{N}$, Adv. Nonlinear Stud. 17 (2017) 677-690.

[41] J. Zhang, J.M. do Ó, M. Squassina, Fractional Schrödinger-Poisson systems with a general subcritical or critical nonlinearity, Adv. Nonlinear Stud. 16 (2016) 15-30.

[42] X. Zhang, B. Zhang, D. Repovš, Existence and symmetry of solutions for critical fractional Schrödinger equations with bounded potentials, Nonlinear Anal. 142 (2016) 48-68.

[43] X. Zhang, B. Zhang, M. Xiang, Ground states for fractional Schrödinger equations involving a critical nonlinearity, Adv. Nonlinear Anal. 5 (2016) 293-314. 